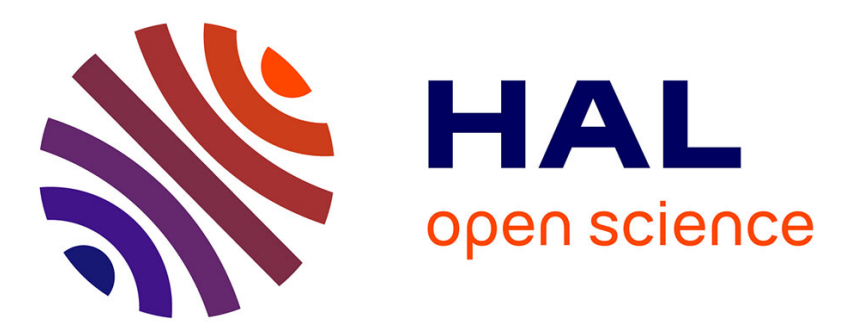

\title{
Photonic and Phononic Band Gap Properties of Lithium Niobate
}

\author{
M.-P. Bernal, M. Roussey, F.I Baida, S. Benchabane, A. Khelif, V. Laude
}

\section{To cite this version:}

M.-P. Bernal, M. Roussey, F.I Baida, S. Benchabane, A. Khelif, et al.. Photonic and Phononic Band Gap Properties of Lithium Niobate. Ferroelectric Crystals for Photonic Applications, Springer, pp.307336, 2009, Springer Series in Materials Science, 10.1007/978-3-540-77965-0_12 . hal-00350057

\section{HAL Id: hal-00350057 https://hal.science/hal-00350057}

Submitted on 17 Apr 2021

HAL is a multi-disciplinary open access archive for the deposit and dissemination of scientific research documents, whether they are published or not. The documents may come from teaching and research institutions in France or abroad, or from public or private research centers.
L'archive ouverte pluridisciplinaire HAL, est destinée au dépôt et à la diffusion de documents scientifiques de niveau recherche, publiés ou non, émanant des établissements d'enseignement et de recherche français ou étrangers, des laboratoires publics ou privés. 


\title{
12 Photonic and Phononic Band Gap Properties of Lithium Niobate
}

\author{
M.P. Bernal, M. Roussey, F. Baida, S. Benchabane, A. Khelif, and V. Laude
}

\subsection{Introduction}

Photonic crystals (PtCs) [1, 2], also known as photonic band gap materials, are attractive optical materials for controlling and manipulating the flow of light. They are of great interest for both fundamental and applied research, and are expected to find commercial applications soon. Their structure consists basically in periodic changes of the dielectric constant on a length scale comparable to optical wavelengths. This periodical modulation of such property can be induced along one, two, or three directions in space. This has similar influence on the propagation of light as atomic crystalline potential has on electrons.

$\mathrm{PtCs}$ in high refractive index contrast semiconductor materials ( $\mathrm{Si}, \mathrm{AsGa}$, etc.) are currently being pursued to obtain a range of forbidden frequencies (i.e. a photonic band gap) in the optical region of the electromagnetic spectrum. A simple example consists of a periodic array of voids within dielectric material. Multiple interference between scattered light waves can eventually lead to some frequencies not being allowed to propagate, giving rise to forbidden and allowed bands, analogous to the electronic bands of a semiconductor. Since the periodicity of the medium must be comparable to the wavelengths of the electromagnetic waves to inhibit their propagation, photonic band gap materials in the optical or infrared domain require sub-micron structures, which can be realized using nano-fabrication technology. The first commercial products involving 2D periodic PCs are already available in the form of photonic crystal fibers. By introducing artificial defects in a host photonic band structure, it is possible to manipulate photons by localizing the electromagnetic states and trap light [3].

Similarly, phononic crystals (PnCs) $[4,5]$ are novel materials that offer exceptional control over phonons, sound and other mechanical waves. Phononic crystals make use of the fundamental properties of waves, such as scattering and interference to create band gaps; ranges of wavelength within which waves cannot propagate through the structure. The existence of structures with complete phononic band gaps has obvious applications. For instance, a phononic crystal will reflect incoming sound waves with frequencies within the gap and can therefore be used as an acoustic insulator. Moreover, the introduction of defects within the structure allows 
sound waves with frequencies in the band gap to be trapped near a point-like defect, or guided along linear defects. Since the discovery of the phononic crystal occurred after the photonic counterpart, there are fewer research groups working in phononic structures but a continuous publication growth on the field can be observed. It should not be unexpected that more and more researchers get involved in phononic crystals also in the following years.

Phononic crystals in the hypersonic regime $[6,7]$ require wavelengths shorter than $10 \mu \mathrm{m}$ that are comparable to optical wavelengths. In addition, the understanding of hypersonic phonons is crucial for many physical phenomena in materials. For example, the interaction between electrons and high frequency phonons determines the efficiency of spontaneous light emission in silicon and other semiconductor materials that have an indirect electronic band gap. Greater control over the phonons in silicon could therefore lead to highly efficient silicon-based light-emitting devices. The challenge in hypersonic crystals concerns the fabrication technology. In contrast to sonic and ultrasonic crystals, which are macroscopic and can be readily made using standard manufacturing techniques, hypersonic crystals require patterns to be created at the submicron and nanometric scales. These challenges are comparable to the ones encountered in photonic crystal fabrication technology.

Lithium niobate $(\mathrm{LN})$ is our material of choice for manufacturing both photonic and phononic crystals. It is indeed well known that monocrystaline LN is a material with many interesting nonlinear properties: it is at once ferroelectric, piezoelectric, electrooptic, photorefractive, and acousto-optic. Its high purity makes it a material of choice for micro wave frequency, optical and surface acoustic wave applications. Though it is a quite chemically insensitive material, and hence quite resilient to traditional etching techniques (as opposed to semiconductor materials such as silicon or gallium arsenide), promising etching techniques are appearing for the achievement of periodic nanostructures with high filling fractions and reasonable aspect ratios. These techniques are furthermore compatible with optical and acoustic surface wave guides. Thus they open the path for the fabrication of two dimensional photonic and phononic crystals where waves are confined in all three dimensions.

This chapter is organized as follows. Section 12.2 is devoted to a presentation of lithium niobate photonic crystals. The theoretical band structures will be used to discuss the appearance of band gaps and of slow light modes. By slow light, we refer to conditions were the group velocity of optical waves is significantly reduced from the usual velocities in homogeneous materials. Then a presentation of actual LN photonic crystals will be made and a striking phenomenon of enhanced electrooptic coefficient will be shown. In Sect. 12.3, phononic crystals fabricated in lithium niobate will be discussed theoretically and experimentally. The emphasis is here on the appearance of phononic band gap properties for surface acoustic waves, i.e. for phonons that are confined close to the surface. It will be shown that despite the fact that the radiation conditions could be expected to lead to highly leaky surface modes, surface modes exist in the phononic crystal where they can be exited. We conclude in Sect. 12.4 by summarizing our results and by giving some perspectives 
in the development of thin layer devices and of phoXonic crystals, artificial crystals that possess simultaneous photonic and phononic band gaps.

\subsection{Photonic Crystals}

\subsubsection{Band Structure Theory and Slow Light}

As is well known, the result of the matter-light interaction greatly depends on the duration of this mutual action. Thereby, the group velocity of the light plays a key role: for a very fast energy propagation, the interaction between light and matter mainly leads to its linear response. To be efficiently excited, non linearities need a very high electromagnetic energy. This last condition can be fulfilled by a local enhancement of the electromagnetic field. Consequently, slow light propagation causes a local exaltation of the electromagnetic field and vice versa.

Lithium niobate, an anisotropic material, has a quite good nonlinear response, especially for the Pockels effect (or electrooptic effect). This effect can be optimized if we consider an $X$-cut substrate to build a PtC. Due to their dispersion properties that can almost be modified as desired, PtCs are most suitable candidates to obtain a light speed reduction. In fact, the main condition to satisfy concerns the dispersion curve of the PtC that must be flat enough for the considered spectral range and according to the desired propagation direction. Based on this choice, we can theoretically determine the PtC geometry assuming that it is made of holes in $\mathrm{LiNbO}_{3}$. For this purpose, we consider and calculate the band diagrams for the most familiar 2D lattices (square, triangular and honeycomb). The dispersion diagram is presented in Fig. 12.1(a) over the irreducible Brillouin zone. A Plane Wave Expansion (PWE) calculation is done for a period over hole radius ratio of 0.27 and a square lattice of air holes engraved in $\mathrm{LiNbO}_{3}$. The extraordinary effective refractive index of the lithium niobate waveguide $\left(n_{\mathrm{e}}\right)$ is considered as the background index. This value is theoretically determined by considering the waveguide in which the $\mathrm{PtC}$ will be fabricated in practice $\left(\varepsilon_{\mathrm{b}}=n_{\mathrm{e}}^{2}=2.143^{2}=4.5924\right)$.

The grey rectangle on Fig. 12.1b emphasizes the zone where a flat dispersion curve is obtained. Theoretically, such eigenmodes can lead to a small group velocity for light propagating inside the $\mathrm{PtC}$ at the corresponding frequency or wavelength. According to the result of Fig. 12.1(b), we choose the $\Gamma X$ direction as the propagation direction and we expect a very large reduction of the group velocity especially for the dashed blue line in Fig. 12.1(c). This last line corresponds to the lower edge (small values of the wavelength) of the second partial Photonic Band Gap (PBG) that will be shown further in Fig. 12.2.

In its general form, the PWE method takes into account an infinite PtC structure. Practically, the size of the $\mathrm{PtC}$ is finite and the band structure is slightly modified. In order to have a more realistic model for the structure, 2D FDTD calculations have been performed. The FDTD algorithm is widely used in the domain of electromagnetism $[8,9]$. It is based on the direct resolution of the Maxwell curl equations by 

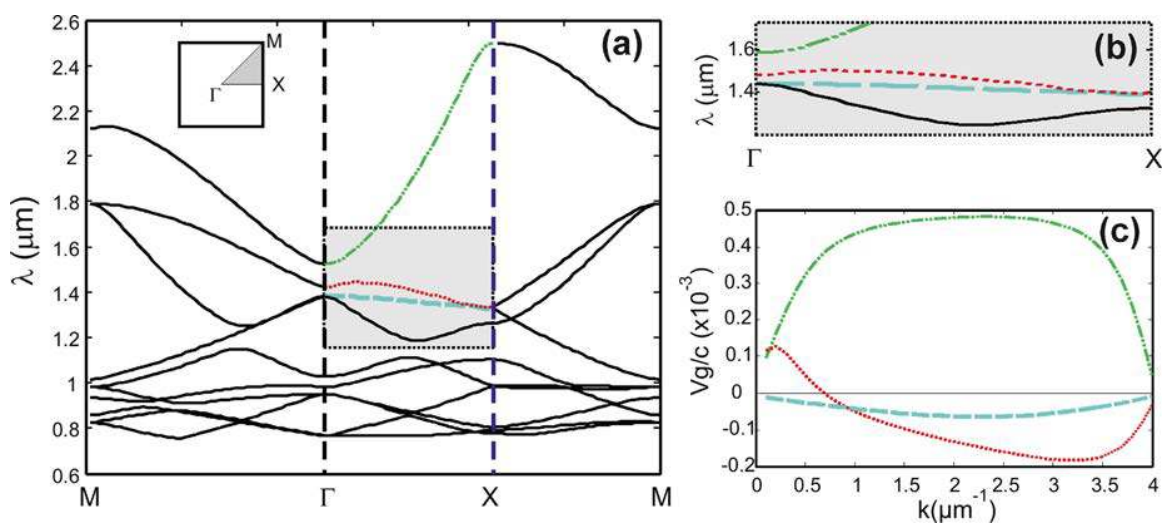

Fig. 12.1. Band diagram of the square lattice of air holes in $\mathrm{LN}$ for $r / a=0.27$ and $a=766 \mathrm{~nm}$ in (a). (b) Is a zoom-in of the dotted rectangular area of (a). It shows a flat dispersion curve that corresponds to modes that provide slow light propagation. (c) Presents the calculated group velocity from (b)

discretizing and then replacing both time and space derivatives by their centered finite differences. The structure is then described by its dielectric permittivity and its magnetic permeability. For LN, which is a dielectric material, we only need to set the permittivity on each node of the spatial grid.

In our home-made FDTD code, we used an orthogonal Cartesian mesh. The resulting staircase effect typically leads to parasitic diffractive nodes that can be reduced by two ways: the first is based on the implementation of a non-uniform mesh in the code [10]. This mesh is based on a gradual variation of the spatial step in order to describe more accurately the fine details of the structure. Consequently, inside the photonic crystal a fine mesh is considered $(\delta=18.683 \mathrm{~nm})$ while a bigger spatial step is used $(\Delta=35 \mathrm{~nm})$ outside the PtC. The second way involves the use of a staggered grid [11]. The conventional cell, corresponding to one period of the $\mathrm{PtC}$, would be defined by a grid of $41 \times 41$ nodes. Nevertheless, this is not sufficient to get a good hole definition. Thus, each point of the previous grid is considered as a cell of $20 \times 20$ points and the dielectric constant of the grid point is taken to be the average dielectric value over these $20 \times 20$ nodes of this sub-grid.

In addition we have introduced the PML technique of Bérenger [12] in order to avoid parasitical reflections on the edges of the computational window. We note that these absorbing boundary conditions (the PML) are not efficient for evanescent waves. Thus, the PML layers are placed at a distance larger than $\lambda_{\max } / 2$ from the outer holes of the structure, $\lambda_{\max }$ being the largest wavelength in the studied spectral area.

The transmission spectrum of a 15 rows long PtC is presented in Fig. 12.2. A propagation along the $\Gamma X$ direction and a TE polarization are assumed. The latter corresponds to the polarization of the experimental guided mode of an annealed proton exchanged (APE) LN waveguide (see Sect. 12.2.2). This spectrum shows 


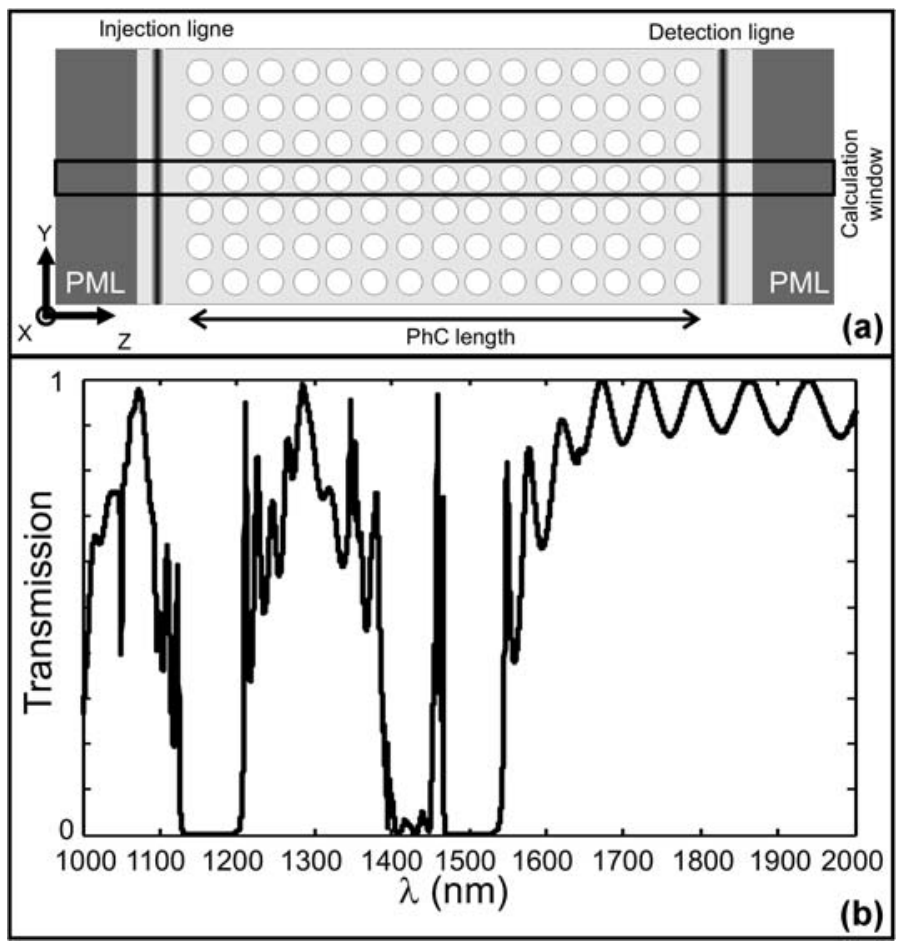

Fig. 12.2. (a) Is the scheme of the calculation window including the PML layers and (b) is the corresponding transmission spectrum of a 15 rows long LN PtC. The modeled PtC is supposed to be infinitely periodic along the transversal direction ( $y$-axis)

three band gaps: the first is centered around $\lambda=1160 \mathrm{~nm}$, the second and the third ones are located between $\lambda=1400 \mathrm{~nm}$ and $\lambda=1550 \mathrm{~nm}$. They are separated by a very sharp transmission peak that is due to the finite size of the PtC [13].

To quantify the enhancement of the Pockels effect induced by nanostructuring, we have to point out and to calculate the local enhancement of the electromagnetic field inside the PtC. For a bulk material, the Pockels effect causes a modification of the refractive index given by:

$$
\Delta n=-\frac{1}{2} \times n_{\mathrm{e}}^{3} \times r_{33} \times E_{\mathrm{s}},
$$

where $r_{33} \simeq 30.8 \mathrm{pm} / \mathrm{V}$ is the highest electro-optic coefficient of LN and $E_{\mathrm{s}}$ is the external applied electric field. For a structured material, this index modification can be expressed by a similar equation:

$$
\Delta n=-\frac{1}{2} \times n_{\mathrm{e}}^{3} \times r_{33} \times f^{3} \times E_{\mathrm{s}},
$$

where $f$ is the local field factor that corresponds to the enhancement of the electromagnetic field due to the nanostructuring of the substrate i.e. to the PtC. $f$ becomes 
here the key parameter that must be optimized in order to enhance the Pockels effect. It can be calculated via the group velocity or via the electric field enhancement inside the $\mathrm{PtC}$ as follows:

$$
\begin{aligned}
f & =\sqrt{\frac{v_{\mathrm{g}}^{\mathrm{BULK}}}{v_{\mathrm{g}}^{\mathrm{PC}}}}, \\
f & =\frac{1}{S} \int_{\mathrm{PC}} \frac{E_{\text {local }}^{\mathrm{PC}}}{E_{\text {local }}^{\text {BULK }} \mathrm{d} y \mathrm{~d} z .}
\end{aligned}
$$

In these equations, $v_{\mathrm{g}}$ is the group velocity of the light inside the bulk material or inside the PtC, $E_{\text {local }}$ is the local optical electric field and $S$ is the surface area of the $\mathrm{PtC}$ structure. It is clear that the local field factor is equal to 1 outside the photonic band gap (PBG) in order to recover the bulk material properties and it must be equal to zero inside the PBG.

Using (12.3) and (12.4) and considering the square lattice studied above, we have theoretically demonstrated a maximum $f$ value of 7 leading to a variation of the refraction index of $\Delta n=0.33$ for an applied external electric field of $E_{\mathrm{s}}=$ $6.15 \mathrm{~V} / \mu \mathrm{m}$. This value has been obtained for the wavelength corresponding to the lower edge $(\lambda=1395 \mathrm{~nm})$ of the PBG. When introducing this modified value of the refractive index in our numerical code, we observe a photonic band gap shift of about $\Delta \lambda \simeq 200 \mathrm{~nm}$.

To confirm the slowing down of light inside the $\mathrm{PtC}$, we have performed an additional numerical experiment that amounts to the observation of a pulse propagation through a 75 rows long $\mathrm{PtC}$ that is infinite towards the $y$-direction. The PtC is illuminated by a plane wave pulse centred on the band gap edge $(\lambda \simeq 1400 \mathrm{~nm})$. The time delay of the pulse is large enough in order to cover a thin spectral interval $(\Delta \lambda=30 \mathrm{~nm})$. A spatial average value over the $z$-component of the Poynting vector versus time is presented in Fig. 12.3. We mainly observe two different zones. The first one corresponds to light propagating with a group velocity approaching the bulk material value (solid white line), it is associated with wavelengths located outside the PBG. The second zone presents a slower light (dashed white line) with an average group velocity $v_{\mathrm{g}} \simeq 6.1 \times 10^{6} \mathrm{~m} / \mathrm{s}$ that corresponds to the wavelengths of the PBG edge. This corresponds to a group velocity reduction by a factor of about 50 .

Experimentally, the PtC is finite both in the $y$ and $z$ directions. Nevertheless, the phenomenon of light enhancement still exists as it is shown in Fig. 12.4 where the modeled structure corresponds exactly to the fabricated device of Sect. 12.2.2. Both the guide and the PtC are taken into account for the 2D FDTD numerical simulation as seen in Fig. 12.4(a). The geometrical parameters were determined from the SEM image presented in Fig. 12.9(a). The light distribution is presented for three different values of the wavelength. The first one (see Fig. 12.4(b)), for $\lambda=$ $1503 \mathrm{~nm}$, corresponds to a zero transmission while the case of a large transmission (see Fig. 12.4(c)) is obtained for $\lambda=1730 \mathrm{~nm}$. The last image (Fig. 12.4(d)) shows the light confinement inside the PtC obtained at the left edge of the PBG namely for $\lambda=1383 \mathrm{~nm}$. This light confinement is at the origin of the enhancement of the non linear effects as it will be demonstrated further. 


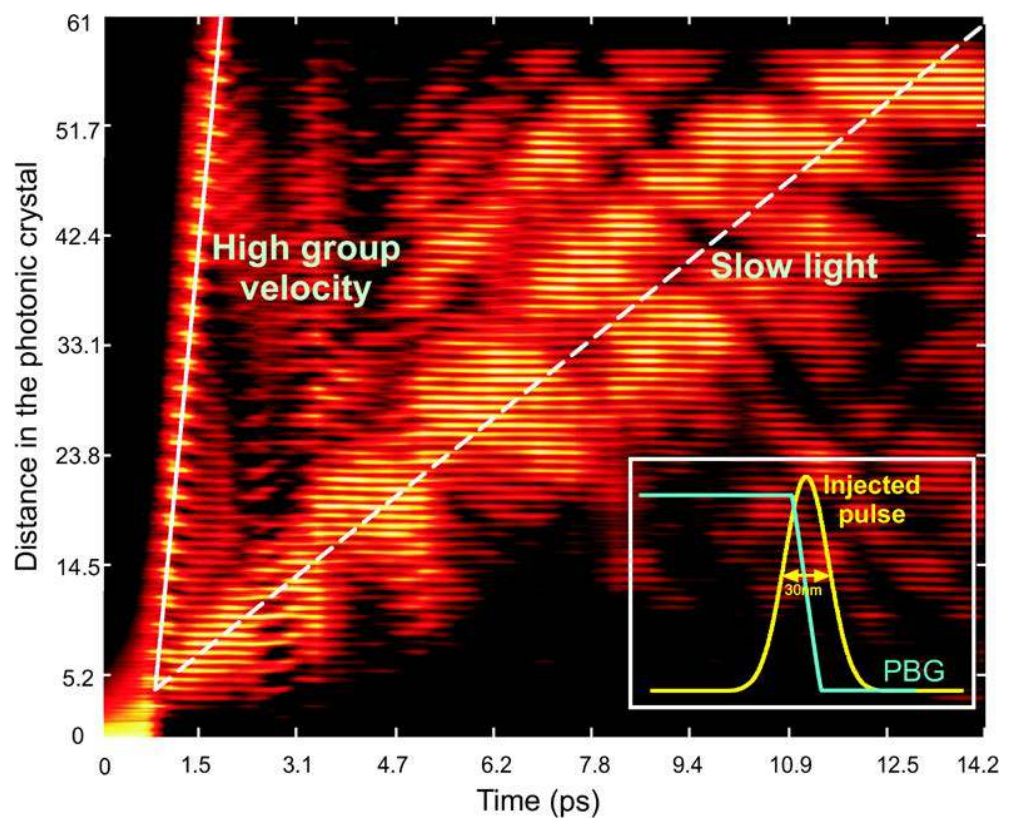

Fig. 12.3. Time evolution of the average value (over one period along the $y$-direction) of the $z$-component of the Poynting vector recorded across the PtC. The injected pulse is shown in the inset; it is centered around the PBG edge and is spectrally large enough to cover the two regimes of transmission (inside and outside the PBG)

\subsubsection{Fabrication and Examples}

Two alternative methods based on focused ion beam bombardement (FIB) to produce photonic band gap structures on $\mathrm{LiNbO}_{3}(\mathrm{LN})$ substrates with a spatial resolution of $70 \mathrm{~nm}$ have been reported. The high resolution and the ability to drill holes directly from the sample surface make FIB milling one of the best candidates for designing good optical quality patterns at submicrometer scale [14]. The only constraint is that the sample surface must be metalized and grounded to avoid charge accumulation. Firstly, we describe the method for directly etching the LN substrate by FIB milling through the metal. This method has been already employed to etch sub-micrometric one-dimensional structures in LN [15]. The second related method is based on RIE etching after FIB milling of the metal layer which behaves as a mask. The advantage of this alternative solution is a lower exposure time. Another expected advantage would be a good replication of the mask shape in the whole hole depth. In both cases, the fabricated submicronic patterns are characterized by FIB imaging. 

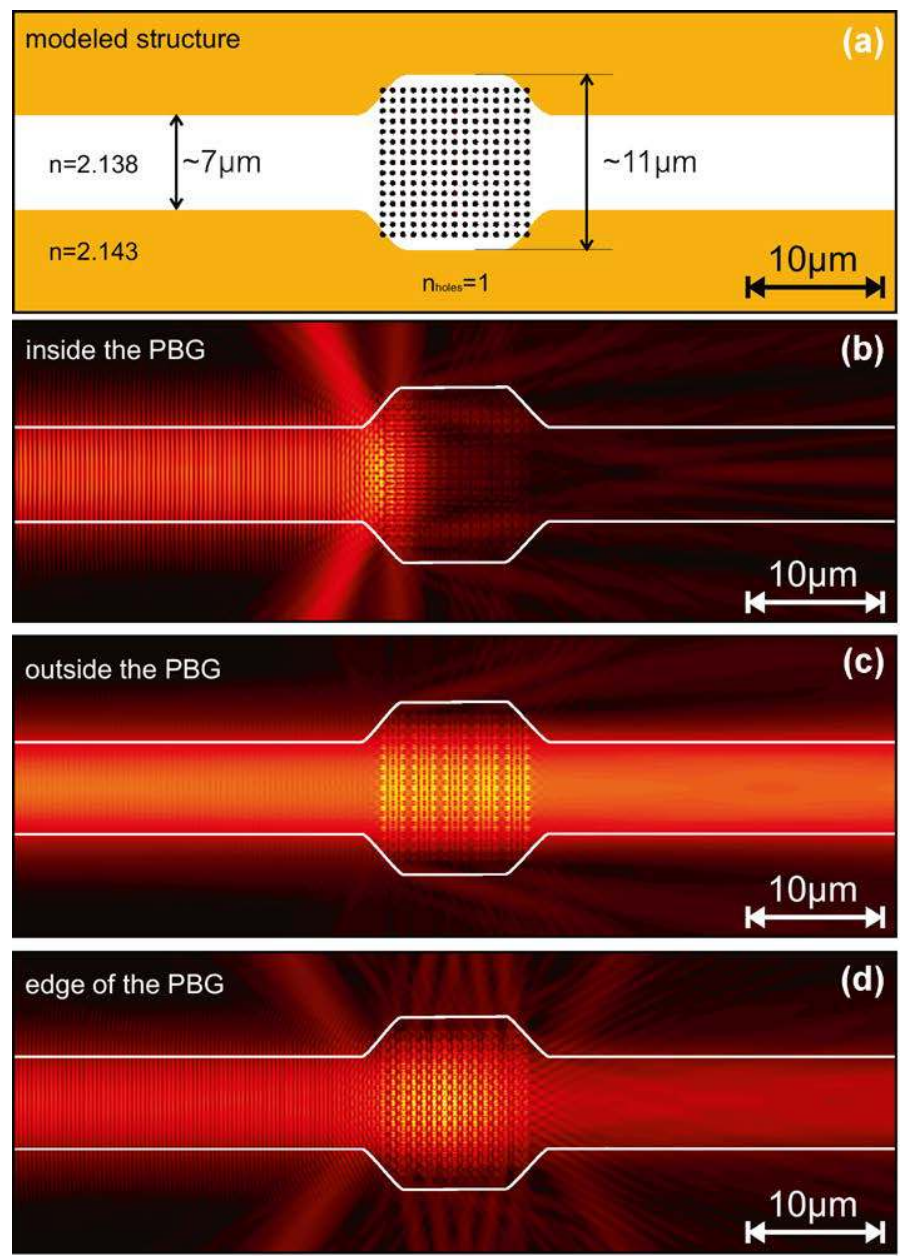

Fig. 12.4. (a) Is the scheme of the calculation window including the waveguide and the PtC; (b), (c) and (d) are the light distribution in color level calculated for three values of the wavelength corresponding to $\lambda=1503 \mathrm{~nm}, \lambda=1730 \mathrm{~nm}$ and $\lambda=1383 \mathrm{~nm}$ respectively

\subsubsection{Experimental Procedure}

The two fabrication processes are schematically shown in Fig. 12.5. The first method, Fig. 12.5(a), is based on a direct etching of the LN substrate by FIB milling. The second one, Fig. 12.5(b), uses the FIB to create the metallic mask and the pattern is then transfered to the LN substrate by RIE. In both cases the sample area is $1 \mathrm{~cm}^{2}$ and the thickness is $500 \mu \mathrm{m}$. A Cr layer is deposited by electron gun evaporation (Balzer, B510) and grounded with a conductive paste before introduction into the FIB vacuum chamber $\left(P=2 \times 10^{-6}\right.$ Torr $)$. In the case of direct FIB writing the 


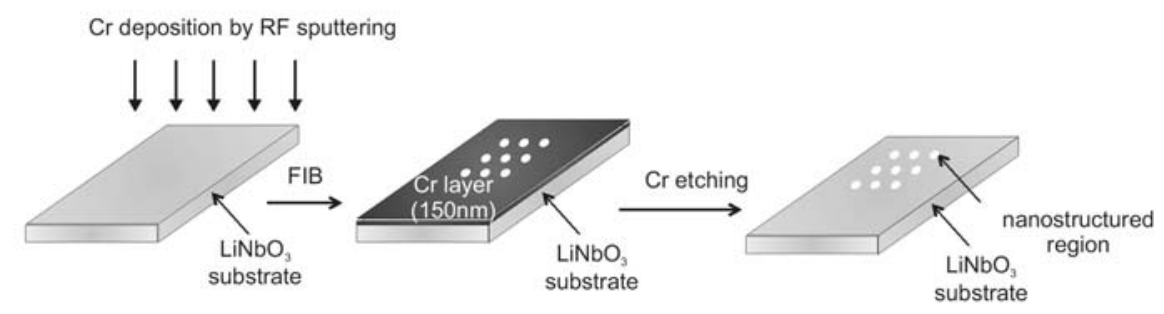

(B)

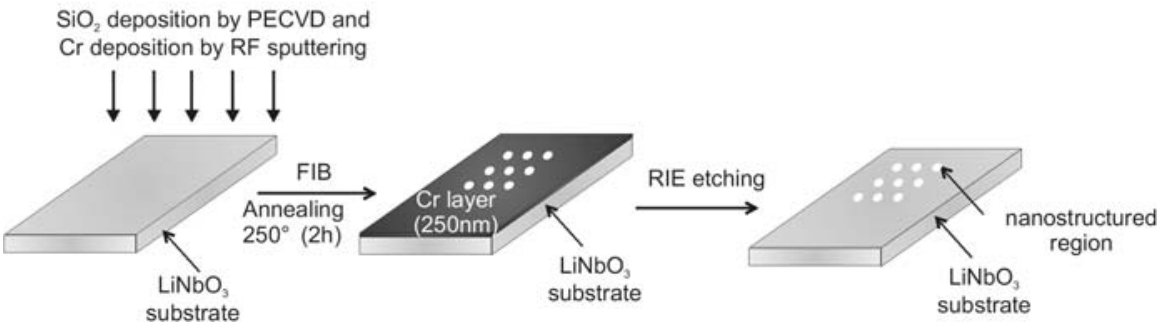

Fig. 12.5. (a) Fabrication steps for lithium niobate nanostructuring using FIB. (b) Fabrication steps for lithium niobate nanostructuring using RIE

thin $\mathrm{Cr}$ metal layer $(150 \mathrm{~nm}$ ) does not modify significantly the etching efficiency. In the second case a thicker $\mathrm{Cr}$ layer $(250 \mathrm{~nm})$ is deposited.

The metal-coated substrates are milled using a focused ion beam column (Orsay Physics - LEO FIB4400 for the case of FIB milling only - Fig. 12.5(a) - and a FEI Dual Beam Strata 235 for the milling of the metallic mask - Fig. 12.5(b)). This method could be directly compared with e-beam lithography. The advantage of FIB patterning of the metallic mask is its ability to selectively remove and deposit material without the use of the additional process step of developing a resist layer.

In the first case (Fig. 12.5(a)) we have fabricated an array of $4 \times 4$ circular holes with $540 \mathrm{~nm}$ diameter and $1 \mu \mathrm{m}$ periodicity. $\mathrm{Ga}^{+}$ions are emitted with a current of $2 \mathrm{pA}$ and accelerated by a voltage of $30 \mathrm{kV}$. The ions are focused with electrostatic lenses on the sample with a probe current of $66 \mathrm{pA}$. The pseudo-Gaussian-shaped spot size is estimated to be $70 \mathrm{~nm}$ on the target. The focused ion beam is scanned on the sample by a computer-controlled deflection field to produce the desired pattern (Elphy Quantum from Raith). A FIB-image cross-section of the cavities is shown in Fig. 12.6. In order to see the etching depth the sample is tilted by $30^{\circ}$ with respect to the FIB axis. As it can be seen from the image, the $4 \times 4$ array exhibits well defined circular holes. The achieved etching depth is approximately $2 \mu \mathrm{m}$ and the etching time was 12 minutes. At $1 \mu \mathrm{m}$ deep the hole diameter is about $432 \mathrm{~nm}$. This conical 


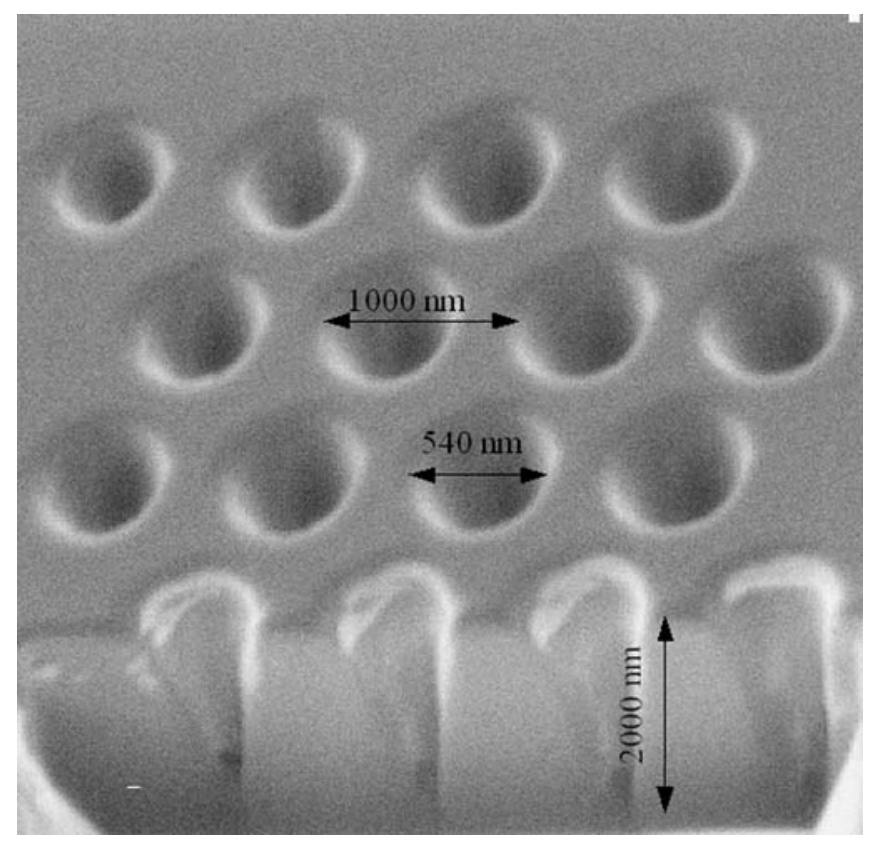

Fig. 12.6. SEM image of a $4 \times 4$ array of holes in lithium niobate etched by FIB

etching shape is due to material redeposition on the sidewalls while milling. In order to reduce the redeposition there are two possible solutions. If the FIB electronics is fast enough (Elphy Quantum is limited to $300 \mathrm{kHz}$ ) and the spot size small enough so that one can scan along the hole sidewalls longer and less on the bottom of the pit.

The second related process requires lower etching time since the desired photonic structure is fabricated at once. In this case, the FIB bombardement is used to pattern a $\mathrm{SiO}_{2}-\mathrm{Cr}$ mask previously deposited on the LN substrate, as depicted in Fig. 12.6(b). The first step consists in depositing a $100 \mathrm{~nm}$ thick layer of $\mathrm{SiO}_{2}$ by Plasma Enhanced Chemical Vapor Deposition (PECVD). A $250 \mathrm{~nm}$ thick chrome layer is then deposited on the substrate by sputtering. The metal is used as a mask for the RIE, while the silica layer prevents the diffusion of Chrome into the substrate during the RIE plasma processing and the increase of the optical losses. This layer is not needed in the case of direct FIB milling since the etching is done locally and the damaged area is defined by the FIB beam size. The samples are annealed at $250^{\circ} \mathrm{C}$ during 2 hours to release stress. The $\mathrm{SiO}_{2}-\mathrm{Cr}$ mask is then nanostructured by FIB patterning, with a current of the sample of $100 \mathrm{pA}$. An exposure time of $3.75 \mathrm{~s}$ is typically required to etch a $250 \mathrm{~nm}$ diameter circular hole, which is 11 times less than the time required in the first process.

The pattern (an array of 24 cylindrical holes) is finally transfered to the substrate by RIE. The relevant parameters of this process are detailed in Table 12.1. It can be noticed that this process requires a very low pressure and a high RF power. In these 
Table 12.1. RIE parameters for the $\mathrm{Z}$ cut of lithium niobate

\begin{tabular}{lllll}
\hline $\begin{array}{l}\text { Pressure } \\
(\mathrm{mBar})\end{array}$ & $\begin{array}{l}\mathrm{SF}_{6} \text { flow } \\
(\mathrm{sccm})\end{array}$ & $\begin{array}{l}\text { RF power } \\
(\text { Watt })\end{array}$ & $\begin{array}{l}\text { Etching rate } \\
(\mathrm{nm} / \mathrm{min})\end{array}$ & $\begin{array}{l}\mathrm{LiNbO}_{3} / \mathrm{Cr} \\
\text { selectivity }\end{array}$ \\
\hline 3 & 10 & 150 & 50 & 0.25 \\
\hline
\end{tabular}

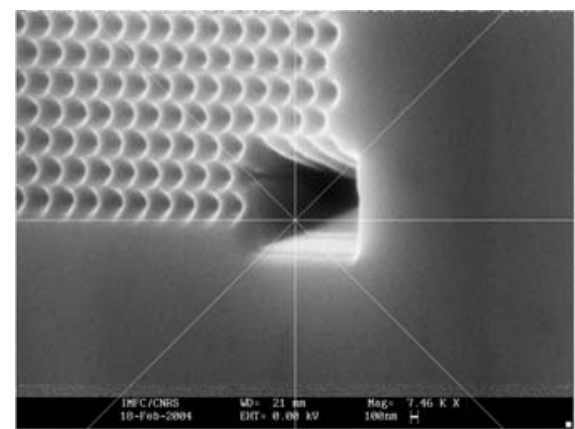

(A)

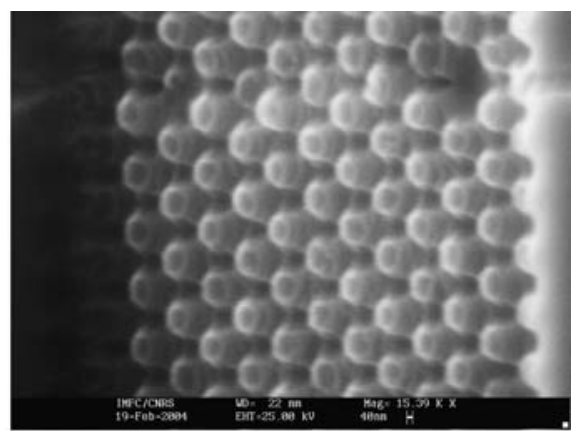

(B)

Fig. 12.7. (a) RIE of holes on a lithium niobate substrate. (b) RIE of pillars on a lithium niobate substrate

conditions, the etch rate of the mask is comparable to the etching rate of the substrate. In order to improve the selectivity of etching between the mask and the LN substrate, we start the process with an exposition of the target to a $\mathrm{O}_{2}$ ionic plasma (pressure $=100 \mu \mathrm{Bar}$, power $=60 \mathrm{~W}$ ). The $250 \mathrm{~nm}$ thick layer of chrome is then more resistant to the $\mathrm{SF}_{6}$-RIE. The selectivity of the mask is thus estimated to be $1: 5$ compared to the LN substrate (while the etching selectivity was measured to be of $1: 2$ without the $\mathrm{O}_{2}$ ionic plasma). The etching rate of the $Z$-cut substrate is measured to be $50 \mathrm{~nm} / \mathrm{min}$. This process is applied to fabricate a triangular lattice of holes with $D=250 \mathrm{~nm}$ and $D=130 \mathrm{~nm}$ diameters and $p=2 D$ periodicity. Figure 12.7(a) and (b) exhibit the SEM images of the holes after FIB milling and 10 min of RIE etching. Figure 12.7(a) shows holes with good reproducibility. The etching depth is measured to be $500 \mathrm{~nm}$. Figure 12.7 (b) shows that the $130 \mathrm{~nm}$ diameter holes were transformed into $130 \mathrm{~nm}$ diameter rods after RIE etching, while the $250 \mathrm{~nm}$ diameter holes were well preserved. This is due to a higher etching rate along the sides of the triangular lattice than in the triangle center when the holes are very close to each other. We can infer from these results that the fabrication of small holes $(D<200 \mathrm{~nm})$ requires lower RF-power to preserve the initial features.

In the next section, three examples of LN photonic crystals are shown. The first example is an hexagonal lattice of air holes in LN. Its photonic band gap (PBG) was experimentally measured. This is the first experimental evidence of a PBG in LN PtCs. The second example shows LN photonic crystals waveguides. Their optical response has been characterize with far field and scanning near field optical microscopy (SNOM). The last example shows an ultra-compact, LN photonic crystal 


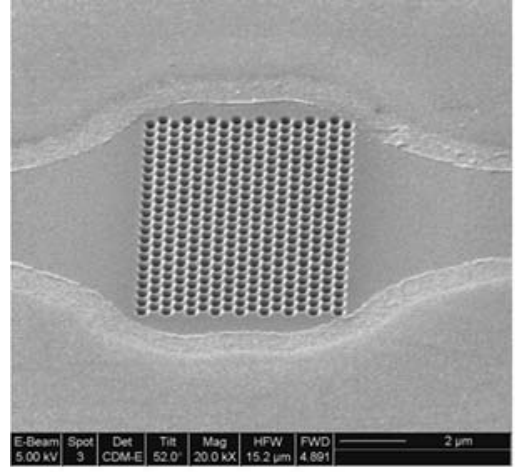

(A)

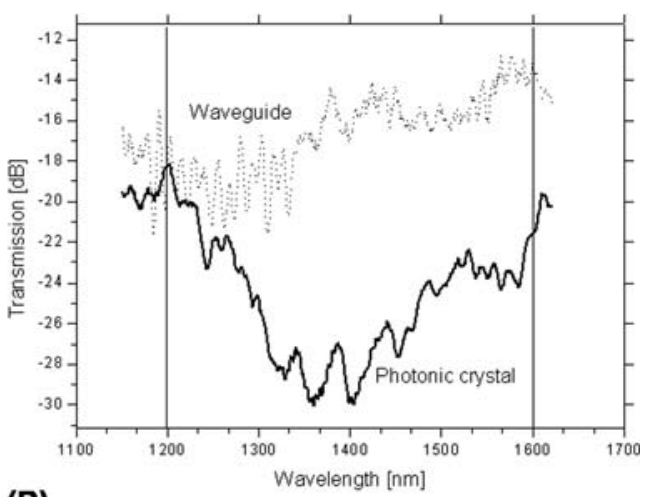

(B)

Fig. 12.8. (a) SEM image of the photonic crystal. (b) Transmission measurement of the photonic crystal

modulator based on the LN electro-optic effect. Its performance (300 times higher than for classical LN modulators) is due to slow light effects as presented previously.

\subsubsection{Measurement of a PBG in a LN Photonic Crystal}

To minimize the optical losses in the vertical direction, the photonic crystal array is fabricated in a lithium niobate channel waveguide. It is already well-known from the literature that in order to have the photonic band gap effect, there must be a maximum interaction between the structure and the guided mode. This requirement is particularly difficult in the case of lithium niobate optical waveguides since the optical mode is very much confined within the substrate. The photonic crystal is fabricated on a $0.3 \mathrm{~mm}$ thick $X$-cut $\mathrm{LiNbO}_{3}$ wafer. In a first step, an optical gradient index waveguide was fabricated by annealed proton exchange (APE). This step was realized through a $\mathrm{SiO}_{2}$ mask in benzoic acid at $180^{\circ} \mathrm{C}$ during 1.5 hours. The process was followed by an annealing of the optical waveguide at $333^{\circ} \mathrm{C}$ for 9 hours. These parameters were chosen to make the optical mode core as close as possible to the surface while keeping single mode propagation at $1.55 \mu \mathrm{m}$. Thus, the mode core is estimated to be at $1.4 \mu \mathrm{m}$ from the surface (much better than the $5 \mu \mathrm{m}$ depth that would be attained with a standard Ti-diffusion process).

The photonic crystal structure was fabricated in the central region on the optical channel waveguide as shown in Fig. 12.8(a). It consists of a hexagonal lattice of $21 \times 19$ circular holes. The holes are fabricated using FIB as described in the previous section.The chosen propagation direction is $\Gamma M$ since theoretical simulations have shown that this direction requires only 15 rows of holes for $100 \%$ extinction ratio [16]. The etching time of the structure $(21 \times 19$ hexagonal hole lattice, hole diameter $=213 \mathrm{~nm}$, periodicity $=425 \mathrm{~nm}$, etching depth $=1500 \mathrm{~nm}$ ) was $20 \mathrm{~min}$. The theoretical transmission band in the $\Gamma M$ direction is $[1300,1600] \mathrm{nm}$.

The transmission spectrum is obtained by coupling a white light fiber source into the channel optical waveguide in which the photonic crystal is located. This su- 


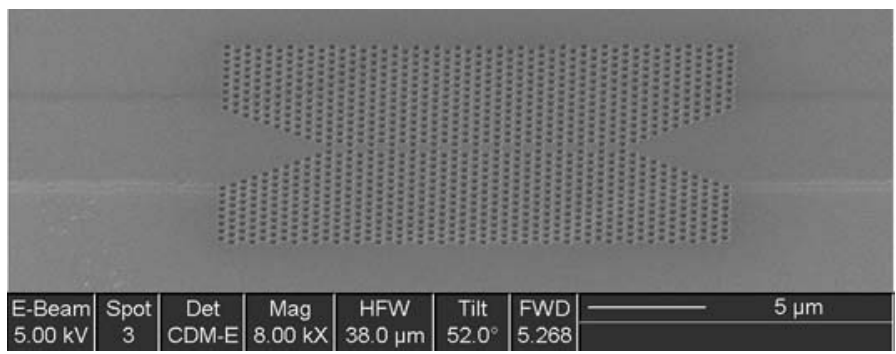

Fig. 12.9. SEM image of a PCW1 fabricated on a lithium niobate substrate with FIB

percontinuum is generated by a sub-nanosecond microchip laser emitting at $532 \mathrm{~nm}$ (mean output power $30 \mathrm{~mW}$, FWHM pulse duration $0.4 \mathrm{~ns}$ ). The polished optical fiber is placed in almost contact to the side entrance wall of the waveguide to decrease the optical input losses. The experimental PBG of the LN photonic crystal is shown in Fig. 12.8(b). The transmission through the photonic array (continuous line) has been compared to the transmission through a standard APE optical waveguide (discontinuous line) fabricated under the same conditions and placed in the same lithium niobate substrate than the photonic array structure. We can see that an extinction ratio of less than $-12 \mathrm{~dB}$ is measured for the case of the photonic crystal structure and that the position of the transmission band corresponds to the theoretical predictions. The noise that is measured in both measurements is mainly due to the fact that the optical waveguide is uniquely monomode at $1.55 \mu \mathrm{m}$ and also due to insertion losses.

\subsubsection{LN PtC Waveguides: Transmission and SNOM Characterization}

In this section, the possibility of guiding the light is experimentally evaluated for photonic crystal waveguides fabricated in $\mathrm{LiNbO}_{3}$. Two alternative structures, based on the same array as in our previous section ( $\Gamma M$ direction) are fabricated. The first one has one line of defects (PCW1), and the second one three lines of defects (PCW3). The etching time of the structures PCW1 and PCW3 $(48 \times 26$ triangular hole lattice, hole diameter $=255 \mathrm{~nm}$, periodicity $=510 \mathrm{~nm}$, etching depth $=1500 \mathrm{~nm}$ ) was $20 \mathrm{~min}$ each. A SEM image of the PCW1 structure is shown in Fig. 12.9.

The novel structures were first characterized by measuring their far field transmission. The light supercontinuum is generated by a sub-nanosecond microchip laser emitting at $1064 \mathrm{~nm}$ with $8 \mu \mathrm{J}$ energy per pulse [17]. The optical transmission was measured through the two photonic waveguides, and through a standard optical waveguide, fabricated on the same wafer and in the same conditions, as described above. The experimental results are shown in Fig. 12.10. As it can be seen in the graph, optical transmissions through the photonic structures (plotted with filled triangles, empty circle, and empty square) exhibit a gap, which does not appear in the transmission through the single APE waveguide (filled circle in Fig. 12.10). In parallel, numerical simulations performed with a commercial software (Bandsolve) of the 


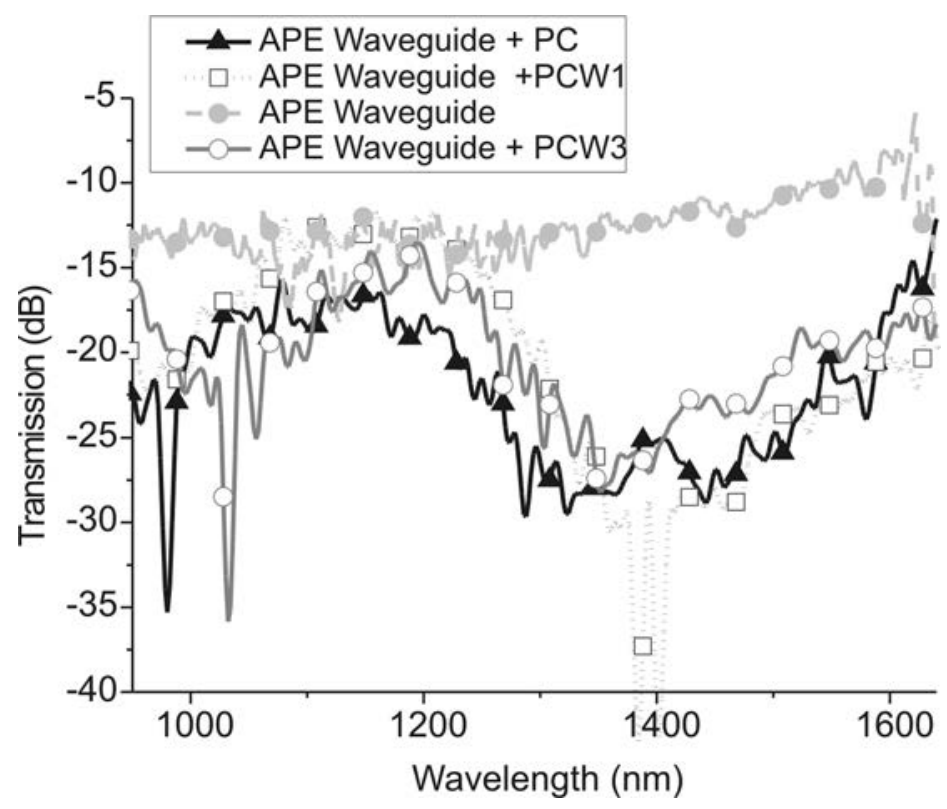

Fig. 12.10. Transmission measurements for APE waveguide only (filled circle), photonic crystal with no defects (filled triangle), PCW1 (empty square), and PCW3 (empty circle)

device without defect lines predict a band gap between $1465 \mathrm{~nm}$ and $1589 \mathrm{~nm}$. The experimental gap starts in a shorter wavelength (approximately around $1300 \mathrm{~nm}$ ) which we believe is a consequence of fabrication imperfections. The dispersion diagram for the case of having one and three defect lines respectively predicts that for the PCW1 case, there is only one mode than is allowed to propagate. As it is intuitive, there is multimodal behavior in the case of a PCW3. Indeed, our simulations show four modes that can propagate within the band gap. Experimentally, the light propagation in the $\mathrm{PtC}$ waveguides is observed by an increase in the transmission inside the gap. This increase is twice more important for the PCW3 case due certainly to the multimodal behavior.

For a deeper interpretation of the propagation of the light through the structures, we have also investigated the near field behavior of the light inside the PtC waveguides. SNOM measurements are relevant in PtCs characterizations because the wave fronts of light in the photonic crystal waveguide undergo substantial modulations on length scales that are much shorter than one wavelength being impossible to resolve the spatial details of light propagation only by the far field transmission measurement described above [18-21].

The instrument used is a commercial scanning near-field optical microscope (SNOM) (NT-MDT SMENA) in collection mode [22] with a dielectric pulled fibred tip. The optical image and topography of the PCW1 at $810 \mathrm{~nm}$ is shown in Fig. 12.11(a) and (b). Figure 12.11(a) shows the topography of the PCW1 structure. The hole depth measured by the SNOM tip is of the order of $30 \mathrm{~nm}$ which is far from 

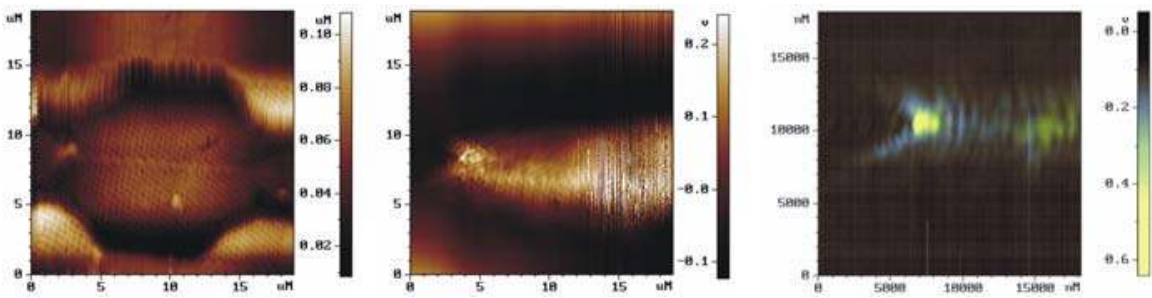

Fig. 12.11. (a) Topography of the photonic crystal structure. (b) SNOM image at $810 \mathrm{~nm}$. (c) SNOM image at $1550 \mathrm{~nm}$

the $1.5 \mu \mathrm{m}$ measured in the SEM image. This is due to the fact that the hole diameter is comparable in size to the tapered fiber, being difficult for the tip to penetrate inside the holes. The signal to noise ratio (SNR) is however high $(\approx 10)$. Figure 12.11(b) shows the optical image of the light going through the PCW1 at $810 \mathrm{~nm}$. As measured by the transmission in Fig. 12.10, the optical transmission is of $-20 \mathrm{~dB}$ since the wavelength is outside the gap of the photonic crystal.

We have also performed the near field measurements in a region inside the gap in which an optical mode propagates $(\lambda \approx 1.55 \mu \mathrm{m}$, transmission $\approx-25 \mathrm{~dB})$. The near field image is show in Fig. 12.11(c). The recorded signal shows clearly a confined mode that propagates through the line of defects. This propagating signal shows a distinct periodicity of $\lambda / n_{\text {eff }}$ obtaining $n_{\text {eff }} \approx 2.2, n_{\text {eff }}$ being the effective index of refraction corresponding to lithium niobate. With these results, we can infer that the step seen in the transmission response of the PCW1 (Fig. 12.10) around $1500 \mathrm{~nm}$ is due to the existence of a guiding region.

\subsubsection{A LN PtC Intensity Modulator}

Tunable PtCs present special interest for integrating dense optical circuits on small surfaces. They typically consist of a periodic array of air holes on a dielectric substrate whose optical properties are modified by an external physical signal (electric or magnetic field, temperature, strain, etc.) [23-26]. One of the most suitable tuning schemes may be based on the application of an electric field, due to the technical compatibility of the $\mathrm{PtC}$ components with current microelectronics technology [27-31]. Indeed, one of the most promising tunable $\mathrm{PtC}$ configurations is a polymerbased photonic device tuned by the Pockels effect showing sub-1 $V$ sensitivity [30]. However, up to now, electro-optical tunable photonic crystals have limited tunability because of the small attainable changes in the refractive index.

The device consists of a $15 \times 15$ square array of air holes etched by Focused Ion Beam (FIB) on a gradient index LN waveguide.The geometrical parameters of the $\mathrm{PtC}$ are fixed by theoretical calculations so that an edge of the gap corresponds to the operating wavelength of $1550 \mathrm{~nm}$. By using two-dimensional Finite Difference Time Domain (FDTD-2D) home-made calculations, we have determined that a squared arrangement of holes with a period $a=766 \mathrm{~nm}$, and $r / a=0.27$ ratio ( $r$ being the radius of the hole) induces a gap with an edge at $1550 \mathrm{~nm}$ in the $\Gamma X$ 

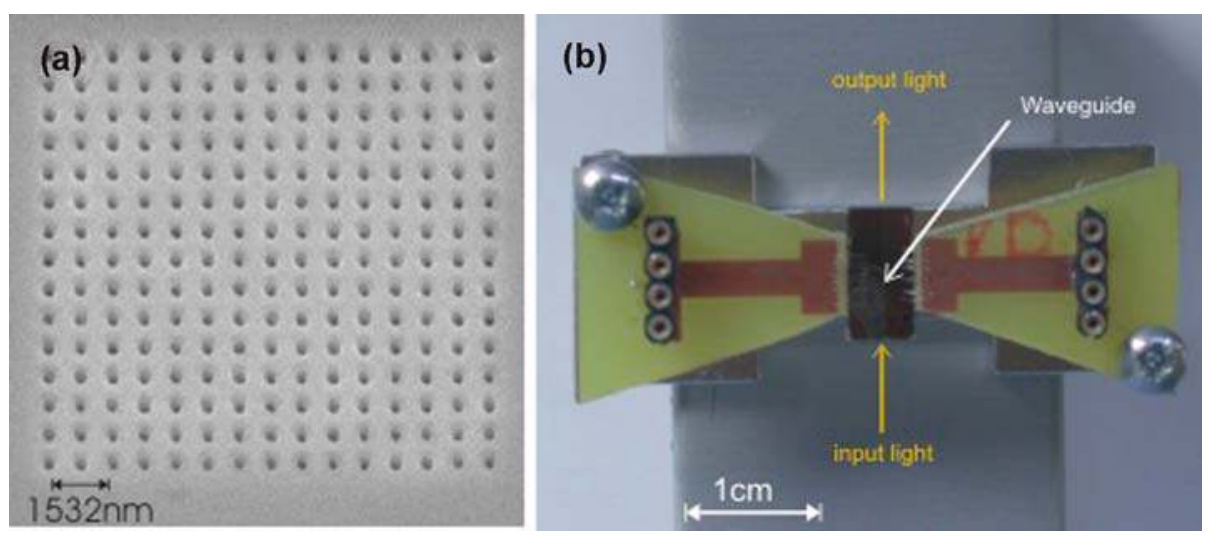

Fig. 12.12. (a) SEM image of the square lattice fabricated by FIB on a LN substrate. (b) Photograph of the final device

direction of propagation and TE polarization. By introducing the classical Pockels effect on the FDTD calculation we can predict an intensity modulation with a $24 \mathrm{~dB}$ extinction ratio for a 0.01 variation of the refractive index at $1550 \mathrm{~nm}$ operating wavelength.

Figure 12.12(a) and (b) show the SEM image of the photonic crystal and a photograph of the fabricated tunable device, respectively. The electrodes have been fabricated by depositing $150 \mathrm{~nm}$ of Ti by sputtering (ALCATEL SCM 450). The distance between both electrodes is $13 \mu \mathrm{m}$ and their length is $8 \mathrm{~mm}$. The LN sample containing the electrodes and the photonic crystal is placed in a butterfly shaped electronic circuit to facilitate the electrical connection. On the circuit, two copper lines have been traced and the Ti electrodes are connected to them through wire bonding.

The transmission spectrum for different excitation voltages is shown in Fig. 12.13. At $0 \mathrm{~V}$, two consecutive stop bands are observed. Their location corresponds to the theoretical prediction (first band $[1125,1200] \mathrm{nm}$, second band $[1400,1550] \mathrm{nm})$ although the measured stop bands are wider due to fabrication imperfections.

When a continuous voltage is applied the band gap shifts. The measured wavelength shift is of $2.5 \mathrm{~nm} / \mathrm{V}$. This value is 312 times bigger than the shift predicted by the Pockels based simulations $(0.008 \mathrm{~nm} / \mathrm{V})$. The band-shift changes direction as we invert the applied voltage sign confirming an electric effect. To rule out photorefractive additional effects we have repeated the same experiment with the sample continuously illuminated by a blue laser beam $(50 \mathrm{~mW}, \lambda=473 \mathrm{~nm})$ over three hours. No additional effect was appreciated. The reasons of this enhancement are explained in Sect. 12.2.1. Indeed, the effective second order susceptibility in the LN nanostructure increases, giving rise to an ultra-compact low voltage $\mathrm{PtC}$ modulator when it operates at its band edge (corresponding to slow photons). 


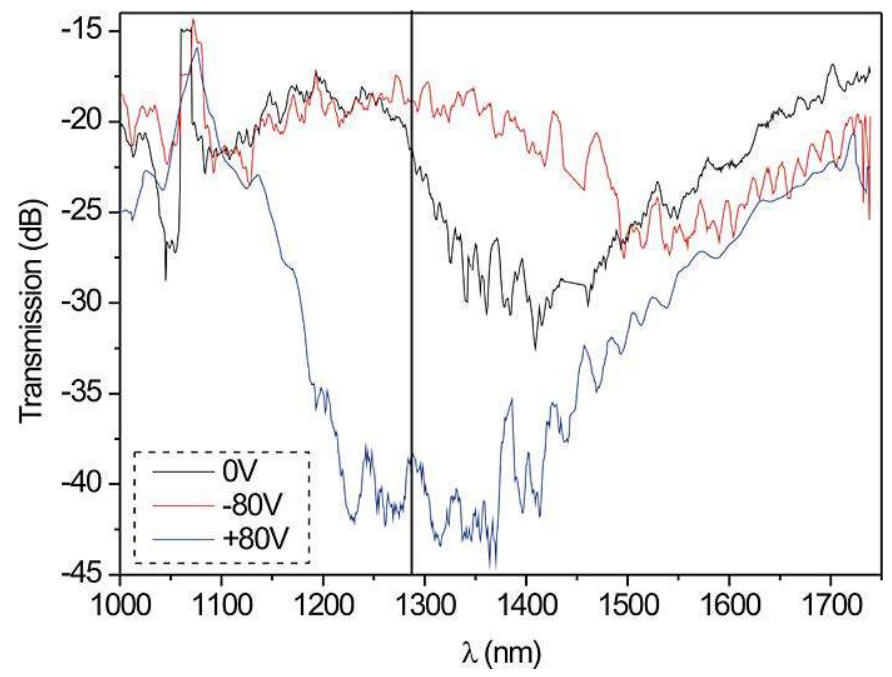

Fig. 12.13. Transmission results at $0 \mathrm{~V},-80 \mathrm{~V}$ and $+80 \mathrm{~V}$

\subsection{Phononic Crystals}

\subsubsection{Theory}

\section{Finite Element Method}

A bulk wave finite element model can be used to calculate phononic crystal band diagrams. This bulk wave approach can be taken as it has been shown by some of the authors elsewhere [32] that band gap position and width for surface waves generally coincides with those for bulk waves, at least in the case of air holes milled in a lithium niobate substrate, as considered here. Although plane wave expansion models have previously been proved to be relevant to predict the band gap position and width by computing the band diagrams of the considered structures, even in the frame of an air/solid phononic crystal, they can appear as rather inaccurate when dealing with extreme geometrical conditions (really high filling fractions, for instance). Finite element methods not only allow to get rid of these limitations, but also present the noticeable advantage of being capable of taking into account the abrupt changes in the propagation conditions that occur at the interface between the matrix material and the inclusions.

The phononic crystal is assumed to be infinite and arranged periodically in the $X$ and $Y$ directions. The whole domain is split into successive unit cells, consisting of a single hole surrounded with the matrix material and indexed by $(m, p)$. The unit cell is meshed and divided into elements connected by nodes. The structure is excited by a plane wave and the incidence is characterized by the real wave vector $\mathbf{k}=\left(k_{x}, k_{y}\right)$. According to the Bloch-Floquet theory, all fields obey a periodicity 
law, yielding for instance the following mechanical displacements $u$ for the $i$ th node:

$$
u_{i}\left(x+m a_{1}, y+p a_{2}, z\right)=u_{i}(x, y, z) \exp \left(-j\left(k_{x} m a_{1}\right)+\left(k_{y} p a_{2}\right)\right),
$$

where $k_{x}$ and $k_{y}$ are the components of the Bloch wavevectors in the $X$ and $Y$ directions respectively and $a_{1}$ and $a_{2}$ are the pitches of the structure. Using this relation allows us to reduce the model to a single unit cell which can be meshed using finite elements using in our case a mechanical displacement and electrical potential formulation scheme. Considering a monochromatic variation of mechanical and electrical fields with a time dependence in $\exp (j \omega t)$ where $\omega$ is the angular frequency, the general piezoelectric problem with no external applied force can then be written as:

$$
\left[\begin{array}{cc}
K_{u u}-\omega^{2} M_{u u} & K_{u \phi} \\
K_{\phi u} & K_{\phi \phi}
\end{array}\right]\left(\begin{array}{l}
u \\
\phi
\end{array}\right)=\left(\begin{array}{l}
0 \\
0
\end{array}\right),
$$

where $K_{u u}$ and $M_{u u}$ correspond to the stiffness and mass matrices of the purely elastic part of the problem, $K_{u \phi}$ is the piezoelectric-coupling matrix, $K_{\phi \phi}$ represents the purely dielectric part and $u$ and $\phi$ are respectively the nodal displacement and electrical potential. As the angular frequency $\omega$ is a periodical function of the wave vector, the problem can be reduced to the first Brillouin zone. The dispersion curves are eventually built by varying the wave vector on the first Brillouin zone for a given propagation direction. The full band diagram is then deduced using the structure symmetries.

\section{Phononic Crystal Design}

Practical interest in the fabrication of phononic crystals usually dwells in the obtaining of the largest possible band gaps. Once the structure type of symmetry is set as a first step, the next critical parameter to take into account is the lattice filling fraction. It has been shown that in an anisotropic and piezoelectric material such as lithium niobate, numerical plane wave expansion simulations predict a fractional band gap width up to $34 \%$ for a $64 \%$ filling fraction for any propagation direction and polarization along the complete anisotropic Brillouin zone [32]. The diameter over pitch $(d / a)$ ratio of the structure is then around 0.9 . Figure 12.14 displays the band diagram obtained for a structure with a different filling fraction, namely 0.94 . Here again, a full band gap clearly opens, with a normalized center frequency $f \times a$ around $200 \mathrm{MHz}$ and a fractional bandwidth of about $35.4 \%$.

If band gap materials with high fractional bandwidths can be obtained in configurations exhibiting high diameter over pitch ratios, crystals with lower $d / a$ values would certainly prove to be easier to fabricate. The point is then to determine how the fractional band gap width scales with the filling fraction of the structure. Figure 12.15 shows this last parameter variation as a function of $d / a$. The band gap width tends to rapidly decrease with the filling fraction of the phononic crystal and is eventually reduced to zero when this latter turns out below $50 \%$, which corresponds to $d / a=0.8$ and does not really allow for a large margin in terms of crystal fabrication. We have hence set our choice on the fabrication of crystals with diameter over pitch ratios higher than 0.9 . 


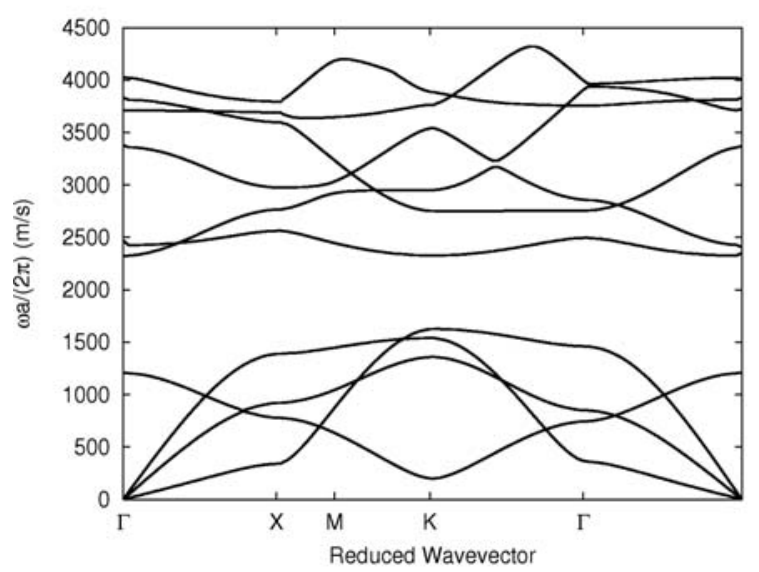

(a)

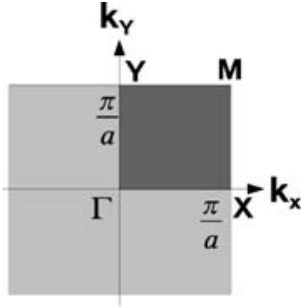

(b)

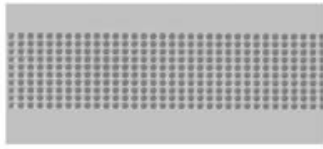

(c)

Fig. 12.14. Theoretical band structure for bulk waves propagating in the plane of a square and void/lithium niobate phononic crystal with a 0.94 diameter over pitch ratio. (b) Sketch of the first Brillouin zone in a square lattice. (c) Scanning Electron Microscope photographs of a $10 \mu \mathrm{m}$ deep, $9.4 \mu \mathrm{m}$ diameter hole etched in a lithium niobate substrate, before removal of the electroplated nickel mask

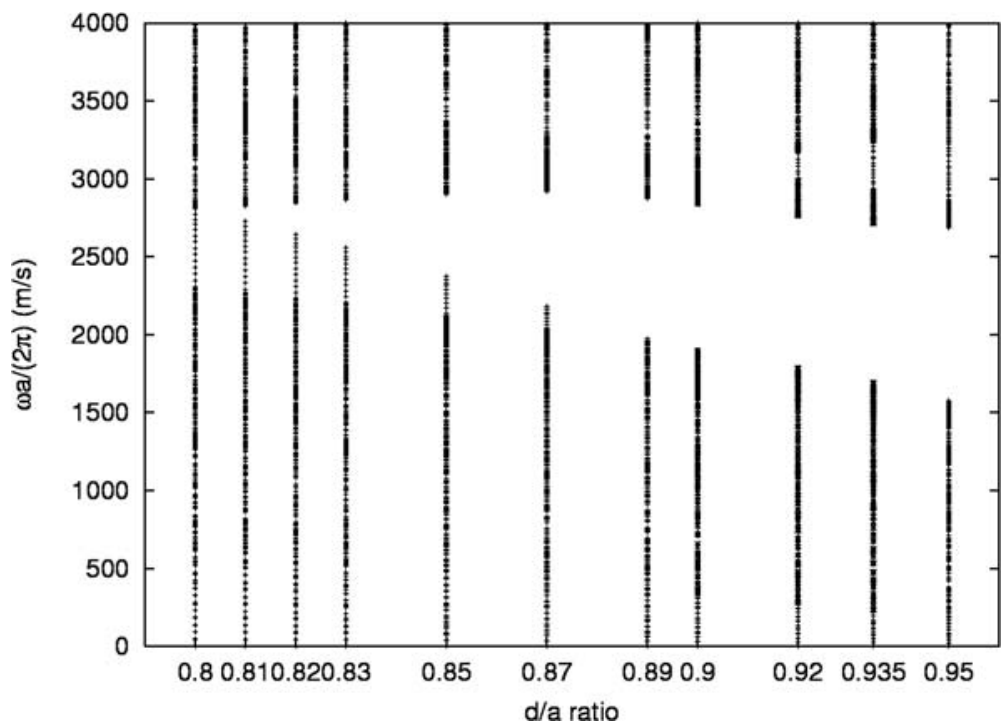

Fig. 12.15. Band gap width versus diameter over pitch ratio for a void/lithium niobate square lattice phononic crystal. The $Y$ cut of lithium niobate is considered 


\subsubsection{Fabrication and Examples}

The phononic crystals have been fabricated on a $500 \mu \mathrm{m}$ thick, $Y$-cut lithium niobate wafer [7]. The material cut has been chosen because of its strong piezoelectric coupling coefficients, however, it is interesting to notice that our fabrication process is quite insensitive to the material cut, and that it is perfectly conceivable to fabricate the same type of phononic crystal in an optical cut of lithium niobate (e.g. the $X$-cut) using the very same fabrication method as developed in the following subsection. To be able to use standard optical lithography for the patterning of the crystal structure, we have chosen to work at a center operating frequency of about $200 \mathrm{MHz}$. This frequency is besides a relevant one in terms of acoustic components operating frequency. This requires hole diameters around $9 \mu \mathrm{m}$ for a $10 \mu \mathrm{m}$ pitch.

\section{Lithium Niobate Etching}

The point was then to mill as deep as possible holes to prevent leakage of surface waves below the crystal. The holes also had to have as vertical walls as possible to avoid surface to volume mode coupling. If this has proven quite easy to achieve in a material such as silicon, where techniques like Deep Reactive Ion Etching are readily available, the problem is more difficult to solve for lithium niobate, as micromachining techniques for this material are far from being well spread. In our case, the wafer has been processed using standard $\mathrm{SF}_{6}$ based Reactive Ion Etching (RIE). The etching rate is considerably low, around $50 \mathrm{~nm} \mathrm{~min}^{-1}$, and the etching slope is quite significant (around $17 \%$ for a $10 \mu \mathrm{m}$ diameter hole). This greatly limits the achievable depth and aspect ratios, as this last parameter seems to be limited to values around 1.5, and creates the need for a particularly selective mask to allow for long etching times. Metallic masks generally satisfy this condition, however, considerably high thicknesses need to be deposited (around $1 \mu \mathrm{m}$ ) to obtain maximum etching depths around $10 \mu \mathrm{m}$. In any case, this etching depth is close to the limit set by the etching slope, but the selectivity of the mask turns out not to be sufficient to ensure a good enough surface state for surface wave transduction, as the process duration generally leads to an almost total depletion of the metallic mask. In this case, the surface of the lithium niobate wafer starts being locally etched, resulting in a deterioration of the overall surface state. The alternative we have chosen is to use a highly selective, $1 \mu \mathrm{m}$ thick electroplated Nickel mask which enables to obtain the $10 \mu \mathrm{m}$ depth we are aiming at with a satisfying hole profile. The mask selectivity would even allow for a deeper etch, which is an interesting result in the frame of applications requiring drilling of larger patterns. The chosen depth of $10 \mu \mathrm{m}$ should anyway remain high enough for surface (Rayleigh) waves to feel the influence of the crystal, due to their limited penetration depth in the substrate. Figure 12.14(c) shows examples of holes obtained in a $Y$-cut lithium niobate wafer before removal of the electroplated Nickel mask. The initial thickness of the mask was around $1 \mu \mathrm{m}$, and one can notice that almost half of this initial thickness is still preserved after the etching process. These figures and further reactive ion etching tests unreported here 
seem to point out that the etching slope increases when the hole diameter decreases which would limit fabrication of higher frequency phononic crystals using the same RIE process. In any case, the surface state inside the holes is highly satisfying.

\section{Crystal Characterization}

Several possibilities are then offered for the characterization of our phononic crystal structure. Optical techniques like Brillouin Light Scattering (BLS), as described for instance in [6] allow for a full retrieval of the considered elastic modes band diagrams. It is also possible to perform phase sensitive measurements which give extensive information on the acoustic field profiles [33, 34]. In our case, and for now, we will limit ourselves to an electrical characterization of the crystal. This latter can be performed by direct excitation and detection of surface acoustic waves traveling through the crystal using for instance, but not necessarily, two identical IDT's, one as a transmitter, and the second one as a receiver. This has the advantage of putting us in a realistic device configuration. However, bandgap measurements produce the need for large bandwidth surface acoustic waves sources, For instance, in the present case, a large frequency range, from 150 to $30 \mathrm{MHz}$ at least, needs to be covered to be able to observe the band gap phenomenon. Simple interdigital transducers unfortunately do not allow to fulfill this requirement. Indeed, increasing the bandwidth of a single IDT can only be done by reducing the number of electrodes of the device. But reducing this number is generally coupled with a loss in the electro-acoustic coupling of the device and with greater interference with bulk waves propagating in the substrate, hence leading to a small dynamic device response. To overcome this problem, a solution proposed in the literature as for instance in [35, 36] and applied in the recent experiments of $\mathrm{Wu}$ and coworkers [37] is to use slanted finger interdigital transducers. However we will see later on that this approach cannot actually be used here. Instead, we here propose an alternative consisting in working with a series of IDT's of varying mechanical period instead. This allows to cover the frequency range of interest by juxtaposing neighboring responses. The main drawback of such a method is that it implies the fabrication of several identical phononic crystals instead of working with a single one. This hence requires some means of "mass-production" of holey structures. This last point can be a limiting factor in some cases but remains fortunately compatible with the optical lithography combined with RIE process described in the previous section. Eight IDT's with ten digit pairs with an emission wavelength ranging from $12.2 \mu \mathrm{m}$ to $26 \mu \mathrm{m}$ were needed to cover the frequency range of interest. The IDT's have been realized by patterning a $150 \mathrm{~nm}$ thick aluminum layer, and oriented for elastic wave propagation along the $\Gamma X, \Gamma M$ and $\Gamma Y$ directions of the first Brillouin zone. Figure 12.16 shows a scanning electron microscope image of some of the phononic crystals for surface acoustic waves manufactured on a single $Y$-cut lithium niobate wafer.

Figure 12.17 displays band diagrams for bulk waves in the $d / a=0.94$ configuration for these three propagation directions. These latter encompass the main points of symmetry of the first Brillouin zone, and are relevant directions to consider 


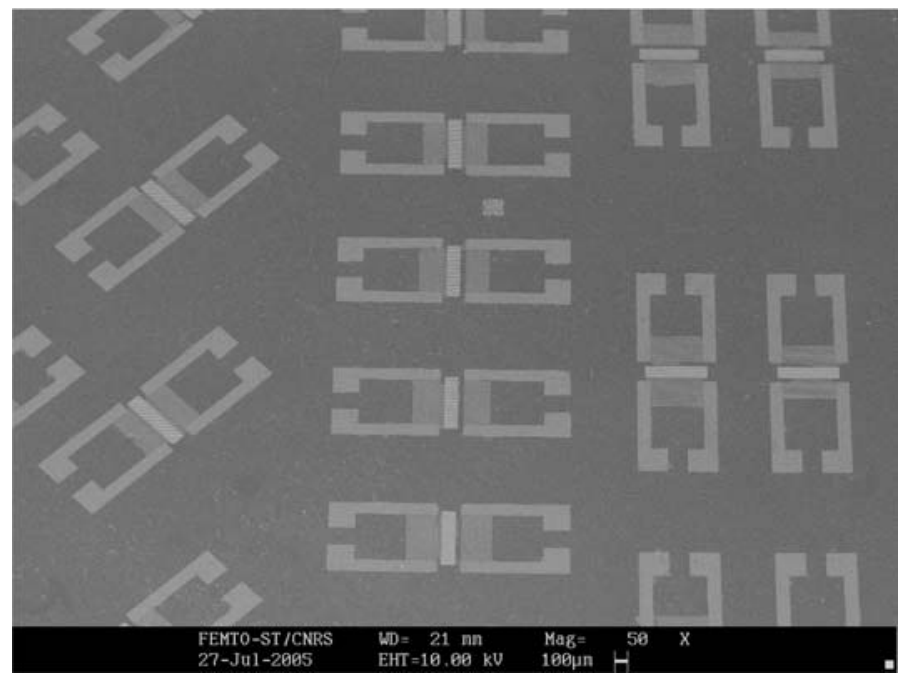

Fig. 12.16. Scanning electron microscope image of some of the phononic crystals for surface acoustic waves manufactured on a single $Y$-cut lithium niobate wafer

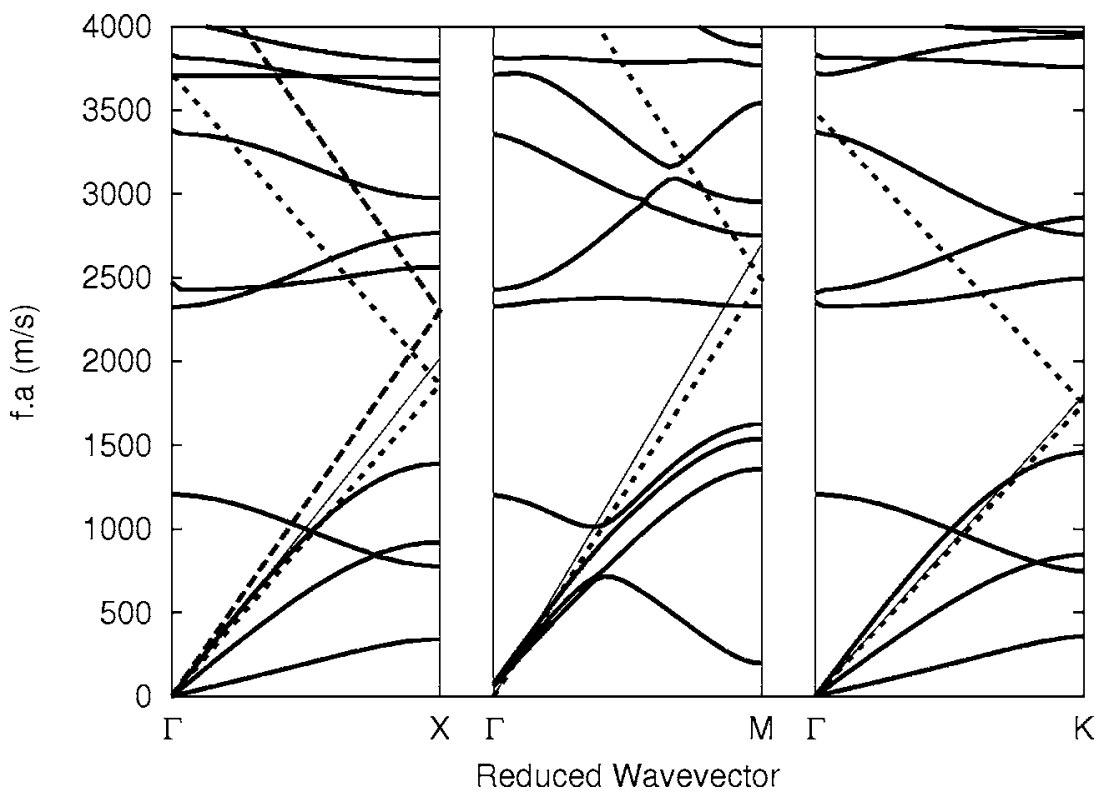

Fig. 12.17. Theoretical band diagrams for bulk waves propagating in the plane of a square lattice void/lithium-niobate phononic crystal with a $69 \%$ filling fraction $(d / a=0.94)$ along the $\Gamma X, \Gamma M$ and $\Gamma Y$ directions of the first Brillouin zone. In the greyed regions, above the soundlines, there is coupling with the radiation modes of the substrate and surface modes become leaky. The dispersion relations on a free surface are also indicated for the Rayleigh (short dashed line) and the leaky (long dashed line) surface waves 

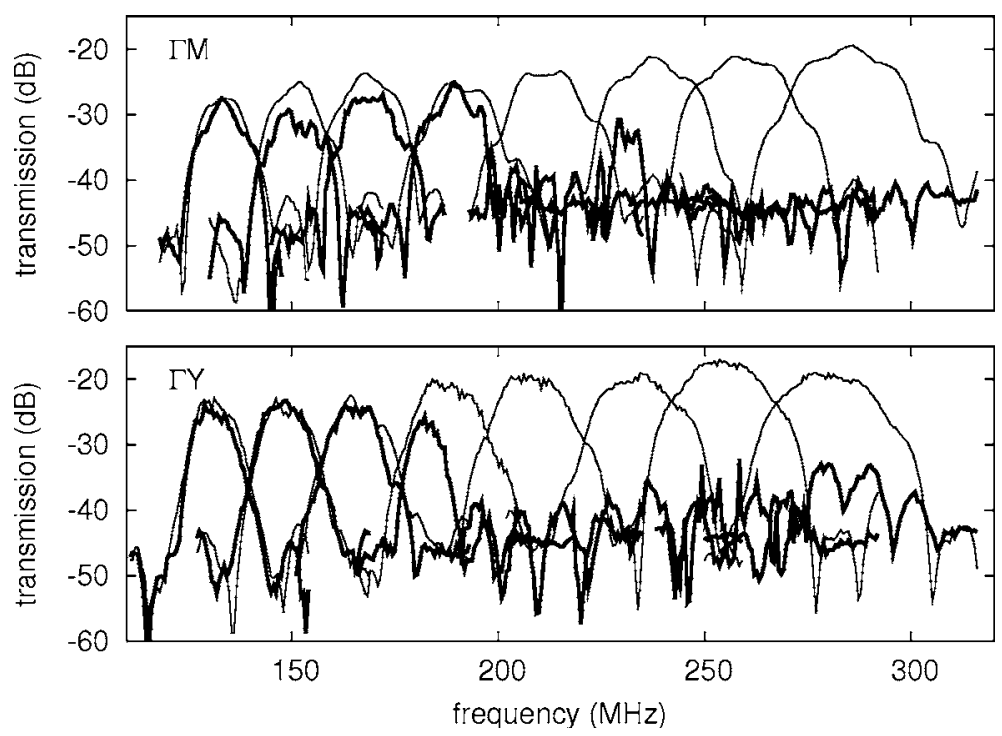

Fig. 12.18. Measurements of the 8 reference devices (dotted line) and the 8 phononic band gap devices (solid line) along the $\Gamma M$ and $\Gamma Y$ directions

in the frame of an experimental investigation of an anisotropic phononic structure. In every case, two series of IDT's have been fabricated, one without any holey structure between the transmitter and the receiver to set a reference, the second one with a phononic crystal as described above. The electrical transmission was then measured in terms of scattering parameters using a network analyzer.

Both transmission ( $S_{12}$ or $S_{21}$, as our device is symmetric) measurements can provide us with relevant information on the Rayleigh waves behavior through the crystal. We expect identical electrical responses for the reference and the phononic devices at frequency below $180 \mathrm{MHz}$ and above $230 \mathrm{MHz}$, which correspond respectively to the lower and upper frequency limits of the theoretically predicted band gap. Between these frequency values, we anticipate an almost complete extinction of the signal. As far as reflexion is concerned, we expect in the same way to get a similar behavior between reference and phononic crystal signal except for an enhanced reflexion phenomenon inside the band gap.

Figure 12.18 displays the transmission spectra for both the reference and the phononic crystal devices along the $\Gamma Y$ (a) and the $\Gamma M$ (b) directions. The dotted lines stand for the reference signal, i.e. for the transmission response of the delay line constituted by two IDT's separated with free space, while the solid lines give the signal transmission through the phononic crystal. In both cases, these measurements clearly show that at frequencies below $180 \mathrm{MHz}$ along $\Gamma Y$, and below $200 \mathrm{MHz}$ along $\Gamma M$, reference and crystal signals almost perfectly overlap: the Rayleigh wave propagation is not affected by the presence of the crystal. Above these frequencies, the transmitted signal experiences a strong attenuation, with losses esti- 
mated to be around $20 \mathrm{~dB}$ for a ten-period long crystal. Surprisingly, there is no increase in transmission for Rayleigh waves at frequencies higher than $230 \mathrm{MHz}$, as it would be expected from the band diagram previously introduced in Fig. 12.14. The signal does not recover at all along $\Gamma Y$, and only a rather narrow transmission peak - about $5 \mathrm{MHz}$ large - around $230 \mathrm{MHz}$ is detected along $\Gamma M$, but there does not seem to be any higher frequency mode propagating in the structure. Any higher frequency mode propagating in the structure seems to be inhibited.

Similar results are observed along the $\Gamma X$ direction. Let us here notice that, in the case of $Y$-cut lithium niobate, in addition to the pure Rayleigh modes aimed at, some leaky surface modes, the so-called pseudo-surface waves (PSAW), also propagate, though their range of existence is limited to angles around the $X$ crystallographic axis. These transversally polarized waves are usually subject to stronger attenuation while propagating, as it is the case along the $\Gamma Y$ and $\Gamma M$ directions previously discussed where their attenuation coefficients are too high to allow for their propagation and detection, but tend on the other hand to penetrate far deeper into the substrate than the pure Rayleigh wave does. This basically means that because of the very limited depth of the holes, and hence of the phononic crystal structure, the pseudo-surface waves are not expected to experience the substrate periodicity in a significant enough way to be attenuated and to give evidence of a band gap effect. Their propagation velocity is here relatively close to the Rayleigh modes velocity (4600 versus $3700 \mathrm{~m} \mathrm{~s}^{-1}$ ), which leads to a partial overlap of the electrical responses of the two types of waves. This makes the use of wide bandwidth slanted fingers IDT's ambiguous for transmission measurements. The overlap between pseudo-surface waves and Rayleigh waves makes it difficult to plot the obtained transmission signals over the whole considered frequency range, as previously done for the two other directions. Thus, for a better readability of the figure, we will hence limit ourselves and present only results obtained for four of the eight IDT's, as shown in Fig. 12.19. The corresponding devices have an emission wavelength of 26, 20.6, 16.4 and $14.6 \mu \mathrm{m}$, respectively. In the case of Fig. 12.19(a) for instance, the first lobe centered around $145 \mathrm{MHz}$ corresponds to the Rayleigh surface wave, while the second lobe, around $170 \mathrm{MHz}$ corresponds to the pseudosurface wave. This latter seems to be slightly affected by the structure, but there is no relevant drop in the transmission value. However, if closer attention is paid to Fig. 12.19(c), which represents a device with an operating frequency located inside the theoretically predicted band gap, while the pseudo-surface wave remains almost unaltered, the Rayleigh wave experiences the same sharp $20 \mathrm{~dB}$ attenuation. Here again, there is no increase in transmission for the Rayleigh wave for a system operating at frequencies higher than the band gap boundary. The cut-off frequency now lies around $190 \mathrm{MHz}$.

Figure 12.20 gives a summary of the obtained measurements. If a full band gap has definitely opened, with the crystal causing a $20 \mathrm{~dB}$ loss in the transmission value for pure surface modes, there does not seem to be any way to retrieve a high amplitude signal at high frequencies. To account for this phenomenon, let us come back to the fact that the actual crystal does not correspond to the ideal, two-dimensional 

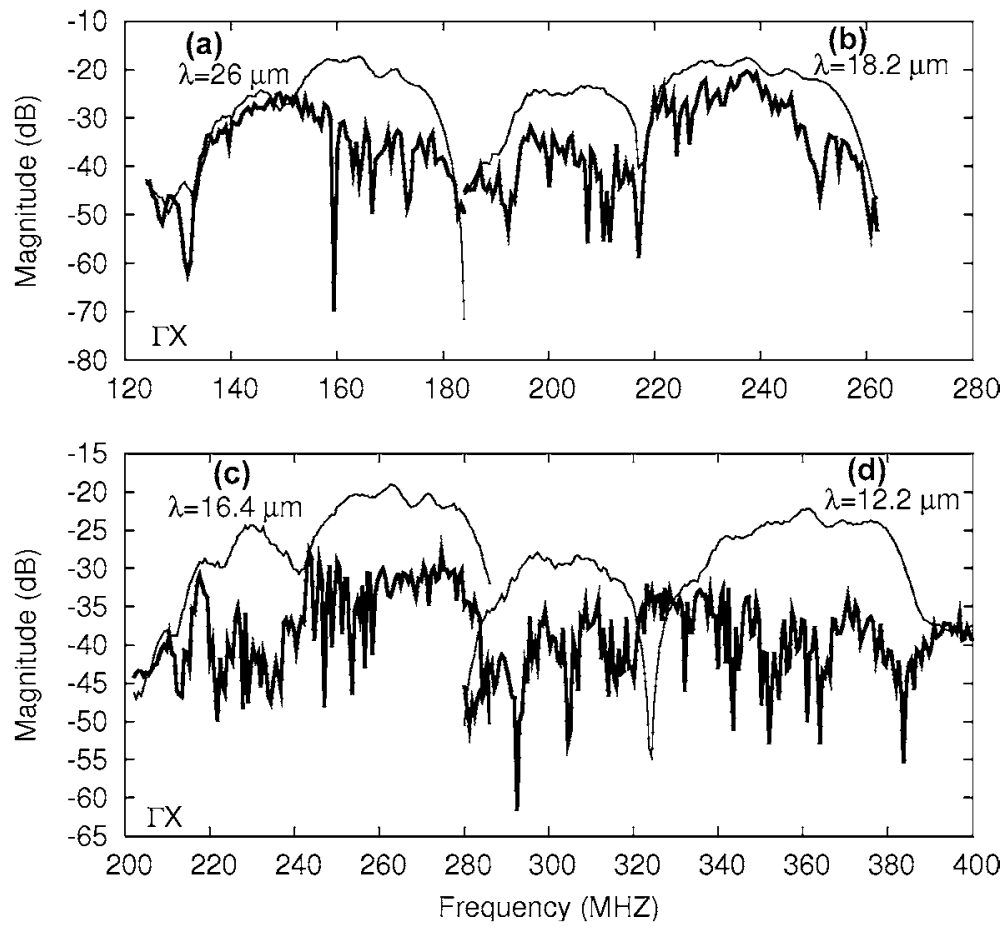

Fig. 12.19. Measurements of 4 reference devices (dotted line) and 4 phononic band gap devices (solid line) along the $\Gamma X$ direction. Both the Rayleigh and the leaky surface waves exist simultaneously and give rise to adjacent frequency responses

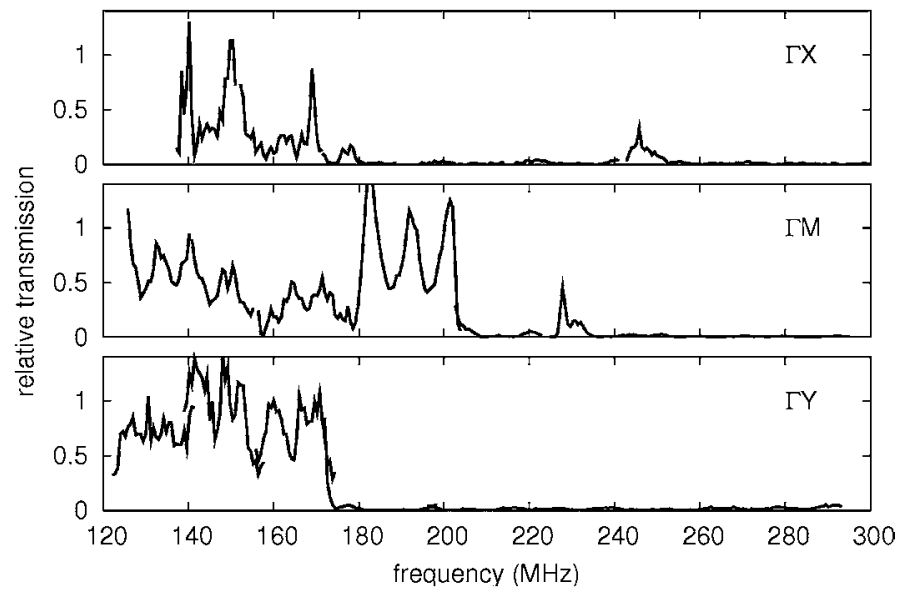

Fig. 12.20. Relative transmission, defined as the ratio of the transmittance with and without a phononic crystal. The complete band gap extends from 203 to $226 \mathrm{MHz}$ 
phononic crystal needed to fully retrieve the theoretically predicted results. The surface waves penetration depth in the substrate remains of the same order, or even larger than the hole depth. The experimental structure can then be seen as a stratified medium, with a void/lithium niobate phononic crystal slab on bulk lithium niobate. This, combined with the conicity of the holes tends to favor out-of-plane scattering, leading to coupling with bulk modes of the substrate, which results in propagation losses for the initial surface waves. To tackle this problem theoretically, an approach quite similar to the one applied to the so-called 2.5D photonic crystals can be taken [3]. These generally consist of a 2D photonic crystal etched through a high-index guiding layer on a lower index substrate. Guided modes as well as radiation modes into the substrate are expected to exist, and the limit between these modes is set by the so-called light line, defined by $\omega=c k$ on the usual $\omega(k)$ dispersion diagram. The straight lines plotted in Fig. 12.17 correspond to the dispersion relation of the bulk modes of the substrate exhibiting the lowest velocity, and which are then the more likely to be coupled into by the scattered surface waves. At frequencies higher than the upper edge of the band gap, the propagating modes lie above this sound line, meaning that they are highly lossy, radiation modes. Only modes below the sound line, i.e. lying at low frequencies can be transmitted, which agrees well with the experimentally observed results. In the case of the Rayleigh surface waves, this sound line accounts for both the total extinction of the signal at high frequencies along the $\Gamma X$ and $\Gamma Y$ directions, but also for the peak observed along $\Gamma M$ which corresponds to modes belonging to the upper part of the band diagram but still lying below this line. The pseudo surface acoustic wave being faster than the slowest shear bulk wave, it is always above the sound line, and hence always experiences strong radiation to the interior of the substrate, in agreement with the experimental observations.

\subsection{Conclusion}

The photonic and phononic band gap properties of lithium niobate were investigated both theoretically and experimentally. The computed photonic band structures were used to discuss the appearance of band gaps and of slow light modes. Actual lithium niobate photonic crystals were presented and a striking phenomenon of enhanced electro-optic coefficient for slow light was emphasized. Phononic crystals fabricated in lithium niobate were then discussed theoretically and experimentally, with an emphasis on phononic band gap properties for surface acoustic waves, i.e. for phonons that are confined close to the surface.

So far, all the research works (from our team and elsewhere) reported on the fabrication of photonic or phononic structures in lithium niobate have been done on bulk material which implies the use of only a small fraction of the surface volume of the material. In the case of LN photonic crystals holes have to be deeply etched in order to reach the optical mode. Optical losses due to inefficient hole-mode interaction and fabrication imperfections could be decreased if the structures were fabricated on thin layers of lithium niobate. Because oxides like lithium niobate tend to 
be chemically very inert, there are only a very limited number of surface modification tools that can be used for fabrication purposes. With the deposition of thin layers of lithium niobate nanostructures will be easily etched or ablated, permitting the fabrication of photolithographically defined two-dimensional and three-dimensional structures on a planar substrate. Some works on epitaxial lithium niobate have been already published in literature [38-40].

Although high-quality films have been fabricated by these techniques, many of the electrical and electro-optical properties reported are generally not comparable to those for bulk single-crystal material. One technique based of the selective Heions implantation called smart cut has been developed mainly by Prof. Osgood at Columbia University and the ETH in Zurich [41-43]. It produces thin layers (of several microns in thickness) of high quality LN. The problem of this technique comes from the technology needed: Implantation doses of $5 \times 10^{16}$ ions $/ \mathrm{cm}^{2}$ are needed. There are only few implantation machines in the world that can provide such a high density.

Once it is realized that despite vastly different frequencies, phonons and photons can share comparable wavelengths, it becomes clear that the simultaneous appearance of photonic (optical waves) and phononic (acoustic waves) band gaps in a single periodic nanostructure can be used to confine simultaneously photons and phonons. This novel phenomenon could have a strong impact on the nature and the strength of photon-phonon interactions. Long term applications include the development of a novel generation of active optical signal processing devices. We name such artificial structures phoXonic crystals. The perspectives of these novel materials are enormous and could lead to breakthroughs in the field of acousto-optics. Appropriately designed, defect structures in deaf and blind structures can lead to simultaneous confinement of light and sound, a result that can have strong influence on photon-phonon interaction and the design of a new class of acousto-optical devices that can integrate the management of elastic and electromagnetic waves. A judicious choice of material is however needed in order to have a simultaneous photonic and phononic band gap. Maldovan et al. [44] have put forward theoretically the use of silicon, a material which has obvious advantages when it comes to etching sub micrometer holes. Provided further technological improvements are made in the direction of nano structuration, we believe that lithium niobate could be a winning option, as the electro-optic and the piezoelectric effects can be exploited with profit in active devices.

\section{Acknowledgments}

The authors are grateful to N. Courjal Bodin for fruitfull discussions and to R. Salut, L. Robert, W. Daniau, J.-Y. Rauch, and G. Ulliac for assistance in the various technological operations. This work was supported by the Action Concerte Incitative under project NANO \#37 COBIAN. 


\section{References}

1. S. John, Strong localization of photons in certain disordered dielectric superlattices. Phys. Rev. Lett. 58(23), 2486-2489 (1987)

2. E. Yablonovitch, Inhibited spontaneous emission in solid-state physics and electronics. Phys. Rev. Lett. 58(20), 2059-2063 (1987)

3. J.D. Joannopoulos, R.D. Meade, J.N. Winn, Photonic Crystals (Princeton University Press, New Jersey, 1995)

4. M.S. Kushwaha, P. Halevi, L. Dobrzynski, B. Djafari-Rouhani, Acoustic band structure of periodic elastic composites. Phys. Rev. Lett. 71(13), 2022-2025 (1993)

5. M.M. Sigalas, E.N. Economou, Band structure of elastic waves in two dimensional systems. Solid State Commun. 86(3), 141-143 (1993)

6. T. Gorishnyy, C.K. Ullal, M. Maldovan, G. Fytas, E.L. Thomas, Hypersonic phononic crystals. Phys. Rev. Lett. 94, 115501 (2005)

7. S. Benchabane, L. Robert, J.-Y. Rauch, A. Khelif, V. Laude, Evidence for complete surface wave band gaps in a piezoelectric phononic crystal. Phys. Rev. E 73, 065601(R) (2006)

8. A. Taflove, S.C. Hagness, Computational Electrodynamics, the Finite-Difference TimeDomain Method, 2nd edn. (Artech House, Norwood, 2005)

9. K. Sakoda, Optical Properties of Photonic Crystals (Springer, Berlin, 2001)

10. J. Seidel, F.I. Baida, L. Bischoff, B. Guizal, S. Grafstrom, D. Van Labeke, L.M. Eng, Coupling between surface plasmon modes on metal films. Phys. Rev. B 69, 121405 (2004)

11. C.T. Chan, Q.L. Yu, K.M. Ho, Order- $n$ spectral method for electromagnetic waves. Phys. Rev. B 51(23), 16635-16642 (1995)

12. J.-P. Berenger, A perfectly matched layer for the absorption of electromagnetic waves. J. Comput. Phys. 114, 185-200 (1994)

13. M. Roussey, F. Baida, M.-P. Bernal, Experimental and theoretical observation of the slow light effect on a tunable photonic crystal. J. Opt. Soc. Am. B 24, 1416 (2007)

14. C.G. Bostan, R.M. de Ridder, V.J. Gadgil, L. Kuipers, A. Driessen, Interactions with a photonic crystal micro-cavity using an AFM in contact or tapping mode operation. In Proceedings Symposium IEE/LEOS Benelux Chapter, IEE/LEOS, Enschede, The Netherlands, p. 25 (2003)

15. S. Yin, Lithium niobate fibers and waveguides: fabrications and applications. Proc. IEEE 87, 1962 (1999)

16. M. Roussey, M.-P. Bernal, N. Courjal, F.I. Baida, Experimental and theoretical characterization of a lithium niobate photonic crystal. Appl. Phys. Lett. 87, 24110 (2005)

17. K.P. Hansen, R.E. Kristiansen, Supercontinuum Generation in Photonic Crystal Fibers. Crystal Fibre (www.crystal-fibre.com)

18. E. Flück, M. Hammer, A.M. Otter, J.P. Korterik, L. Kuipers, N.F. van Hulst, Amplitude and phase evolution of optical fields inside periodic photonic structures. J. Lightwave Technol. 21, 1384 (2003)

19. S.I. Bozhevolnyi, V.S. Volkov, T. Sondergaard, A. Boltasseva, P.I. Borel, M. Kristensen, Near-field imaging of light propagation in photonic crystal waveguides: explicit role of Bloch harmonics. Phys. Rev. B 66, 235204 (2002)

20. H. Gersen, T.J. Karle, R.J.P. Engelen, W. Bogaerts, J.K. Korterik, N.F. van Hulst, T.F. Krauss, L. Kuipers, Real-space observation of ultraslow light in photonic crystal waveguides. Phys. Rev. Lett. 94, 073903 (2005) 
21. D. Gérard, L. Berguiga, F. de Fornel, L. Salomon, C. Seassal, X. Letartre, P. Rojo-Romeo, P. Viktorovitch, Near-field probing of active photonic-crystal structures. Opt. Lett. 27, 173 (2002)

22. D.W. Pohl, Optical near-field scanning microscope. US patent number 4604520 (1986)

23. H. Takeda, Yoshino, Tunable refraction effects in two-dimensional photonic crystals utilizing liquid crystals. Phys. Rev. E 69, 016605 (2004)

24. Y. Jiang, W. Jiang, L. Gu, X. Chen, R.T. Chen, 80-micron interaction length silicon photonic crystal waveguide modulator. Appl. Phys. Lett. 87, 221105 (2005)

25. S.L. Kuai, G. Bader, P.V. Ashrit, Tunable electrochromic photonic crystals. Appl. Phys. Lett. 86, 221105 (2005)

26. D. McPhail, M. Straub, M. Gu, Electrical tuning of three-dimensional photonic crystals using polymer dispersed liquid crystals. Appl. Phys. Lett. 86, 051103 (2005)

27. M. Schmidt, M. Eich, U. Huebner, R. Boucher, Electro-optically tunable photonic crystals. Appl. Phys. Lett. 87, 121110 (2005)

28. H.M.H. Chong, R.M. De La Rue, Tuning of photonic crystal waveguide microcavity by thermooptic effect. IEEE Photonics Technol. Lett. 16, 1528 (2004)

29. M.T. Tinker, J.B. Lee, Thermo-optic photonic crystal light modulator. Appl. Phys. Lett. 86, 221111 (2005)

30. W. Park, J.B. Lee, Mechanically tunable photonic crystal structure. Appl. Phys. Lett. 85, 4845 (2004)

31. C.W. Wong, P.T. Rakich, S.G. Johnson, M. Qi, H.I. Smith, E.P. Ippen, L.C. Kimerling, Y. Jeon, G. Barbastathis, S.-G. Kim, Strain-tunable silicon photonic band gap microcavities in optical waveguides. Appl. Phys. Lett. 84, 1242 (2004)

32. V. Laude, M. Wilm, S. Benchabane, A. Khelif, Full band gap for surface acoustic waves in a piezoelectric phononic crystal. Phys. Rev. E 71, 036607 (2005)

33. R.L. Jungerman, J.E. Bowers, J.B. Green, G.S. Kino, Fiber optic laser probe for acoustic wave measurements. Appl. Phys. Lett. 40, 313-315 (1982)

34. J.V. Knuuttila, P.T. Tikka, M.M. Salomaa, Scanning Michelson interferometer for imaging surface acoustic wave fields. Opt. Lett. 25(9), 613 (2000)

35. C.K. Campbell, Y. Ye, J.J. Sferazza, Wide-band linear phase SAW filter design using slanted transducer fingers. IEEE Trans. Sonics Ultrason. 29, 224 (1982)

36. H. Yatsuda, Y. Takeuchi, S. Yoshikawa, New design techniques for SAW filters using slanted-finger IDTs. In IEEE Ultr. Symp. (1990), p. 61

37. T. Wu, L. Wu, Z. Huang, Frequency band-gap measurement of two-dimensional air/silicon phononic crystals using layered slanted finger interdigital transducers. J. Appl. Phys. 97, 094916 (2005)

38. A.A. Werberg, H.J. Gysling, A.J. Filo, T.N. Blanton, Epitaxial growth of lithium niobate thin films from a single-source organometallic precursor using metalorganic chemical vapour deposition. Appl. Phys. Lett. 62, 946 (1993)

39. R.S. Feigelson, Epitaxial growth of lithium niobate thin films by the solid source MOCVD method. J. Crystal Growth 166, 1 (1996)

40. Y. Shibata, K. Kaya, K. Akashi, M. Kanai, T. Kawai, S. Kawai, Epitaxial growth and surface acoustic wave properties of lithium niobate films grown by pulsed laser deposition. J. Appl. Phys. 77, 1498 (1995)

41. M. Levy, R.M. Osgood, R. Liu, L.E. Cross, G.S. Cargill III, A. Kumar, H. Bakhru, Fabrication of single-crystal lithium niobate films by crystal ion slicing. Appl. Phys. Lett. 73, 2298 (1998)

42. A.M. Radojevic, M. Levy, R.M. Osgood, A. Kumar, H. Bakhru, C. Tian, C. Evans, Large etch-selectivity enhancement in the epitaxial liftoff of single-crystal $\mathrm{LiNbO}_{3}$ films. Appl. Phys. Lett. 74, 3197 (1999) 
43. P. Rabiei, P. Gunter, Optical and electro-optical properties of submicrometer lithium niobate slab waveguides prepared by crystal ion slicing and wafer bonding. Appl. Phys. Lett. 85, 4603 (2004)

44. M. Maldovan, E.L. Thomas, Simultaneous localization of photons and phonons in twodimensional periodic structures. Appl. Phys. Lett. 88, 251907 (2006) 